\title{
Developing an Empirical Model for Jet-Surface Interaction Noise
}

\section{Clifford A. Brown}

Glenn Research Center, Cleveland, Ohio 


\section{NASA STI Program . . . in Profile}

Since its founding, NASA has been dedicated to the advancement of aeronautics and space science. The NASA Scientific and Technical Information (STI) program plays a key part in helping NASA maintain this important role.

The NASA STI Program operates under the auspices of the Agency Chief Information Officer. It collects, organizes, provides for archiving, and disseminates NASA's STI. The NASA STI program provides access to the NASA Aeronautics and Space Database and its public interface, the NASA Technical Reports Server, thus providing one of the largest collections of aeronautical and space science STI in the world. Results are published in both non-NASA channels and by NASA in the NASA STI Report Series, which includes the following report types:

- TECHNICAL PUBLICATION. Reports of completed research or a major significant phase of research that present the results of NASA programs and include extensive data or theoretical analysis. Includes compilations of significant scientific and technical data and information deemed to be of continuing reference value. NASA counterpart of peer-reviewed formal professional papers but has less stringent limitations on manuscript length and extent of graphic presentations.

- TECHNICAL MEMORANDUM. Scientific and technical findings that are preliminary or of specialized interest, e.g., quick release reports, working papers, and bibliographies that contain minimal annotation. Does not contain extensive analysis.

- CONTRACTOR REPORT. Scientific and technical findings by NASA-sponsored contractors and grantees.
- CONFERENCE PUBLICATION. Collected papers from scientific and technical conferences, symposia, seminars, or other meetings sponsored or cosponsored by NASA.

- SPECIAL PUBLICATION. Scientific, technical, or historical information from NASA programs, projects, and missions, often concerned with subjects having substantial public interest.

- TECHNICAL TRANSLATION. Englishlanguage translations of foreign scientific and technical material pertinent to NASA's mission.

Specialized services also include creating custom thesauri, building customized databases, organizing and publishing research results.

For more information about the NASA STI program, see the following:

- Access the NASA STI program home page at http://www.sti.nasa.gov

- E-mail your question to help@sti.nasa.gov

- Fax your question to the NASA STI Information Desk at 443-757-5803

- Phone the NASA STI Information Desk at 443-757-5802

- Write to: STI Information Desk NASA Center for AeroSpace Information 7115 Standard Drive Hanover, MD 21076-1320 
NASA/TM-2014-218120

AIAA-2014-0878

\title{
Developing an Empirical Model for Jet-Surface Interaction Noise
}

\author{
Clifford A. Brown \\ Glenn Research Center, Cleveland, Ohio
}

Prepared for the

SciTech 2014

sponsored by the American Institute of Aeronautics and Astronautics

National Harbor, Maryland, January 13-17, 2014

National Aeronautics and

Space Administration

Glenn Research Center

Cleveland, Ohio 44135 


\section{Acknowledgments}

This work was supported by the NASA Fundamental Aeronautics Program, Fixed Wing and High Speed.

Trade names and trademarks are used in this report for identification only. Their usage does not constitute an official endorsement, either expressed or implied, by the National Aeronautics and Space Administration.

This work was sponsored by the Fundamental Aeronautics Program at the NASA Glenn Research Center.

Level of Review: This material has been technically reviewed by technical management.

Available from

NASA Center for Aerospace Information 7115 Standard Drive

Hanover, MD 21076-1320
National Technical Information Service 5301 Shawnee Road Alexandria, VA 22312

Available electronically at http://www.sti.nasa.gov 


\title{
Developing an Empirical Model for Jet-Surface Interaction Noise
}

\author{
Clifford A. Brown \\ National Aeronautics and Space Administration \\ Glenn Research Center \\ Cleveland, Ohio 44135
}

\begin{abstract}
The process of developing an empirical model for jet-surface interaction noise is described and the resulting model evaluated. Jet-surface interaction noise is generated when the high-speed engine exhaust from modern tightly integrated or conventional high-bypass ratio engine aircraft strikes or flows over the airframe surfaces. An empirical model based on an existing experimental database is developed for use in preliminary design system level studies where computation speed and range of configurations is valued over absolute accuracy to select the most promising (or eliminate the worst) possible designs. The model developed assumes that the jet-surface interaction noise spectra can be separated from the jet mixing noise and described as a parabolic function with three coefficients: peak amplitude, spectral width, and peak frequency. These coefficients are fit to functions of surface length and distance from the jet lipline to form a characteristic spectra which is then adjusted for changes in jet velocity and/or observer angle using scaling laws from published theoretical and experimental work. The resulting model is then evaluated for its ability to reproduce the characteristic spectra and then for reproducing spectra measured at other jet velocities and observer angles; successes and limitations are discussed considering the complexity of the jet-surface interaction noise versus the desire for a model that is simple to implement and quick to execute.
\end{abstract}

\section{Introduction}

M ODERN aircraft designs may employ complex geometry exhaust systems to maximize efficiency or reduce noise. Subsonic aircraft, for example, have benefited from the reduced noise and improved fuel efficiency offered by the current high bypass ratio engines. These larger engines, however, have pushed the exhaust closer to the airframe surfaces where interactions between the exhaust gases and the hard surfaces may create a new noise problem: jet-surface interaction noise. Supersonic aircraft, in an effort to minimize sonic boom, have moved to embedded engine designs which often exhaust gases over aft airframe surfaces creating a similar noise problem, and while the performance impact of these configurations is fairly well understood, the noise implications remain difficult to predict.

A system level study incorporating many aircraft components combines predictions of performance, efficiency, and noise until the optimal design is determined. However, the ability to predict the noise created or shielded when a high-speed engine exhaust interacts with an airframe surface has been, historically, limited. Physics based exhaust noise prediction methods, that incorporate nearby surfaces, are not yet a viable for for optimization design studies over a large parameter space due to either limiting assumptions or long solution times. Acoustic analogies, for example, rely on Reynolds-Averaged-Navier-Stokes computations, which themselves are time consuming, for information about the flow generally assume that the jet is in free-space. More recent predictions using Large Eddy Simulations may include a surface but require far too much time for a system level study (especially if the surface boundary layer must be resolved). However, an empirical model based on available experimental data can offer a first-order approximation of the affect aircraft surfaces near the engine exhaust have on the overall noise levels within the experimental variable space and with a rapid solution time.

An extensive database of jet-surface interaction noise measurements over several tests conducted at the NASA Glenn Research Center's Aero-Acoustic Propulsion Laboratory and supported by both the Fixed 
Wing and High Speed Projects under the Fundamental Aeronautics Program. These far-field noise data form the basis for an empirical model capable of describing the surface trailing edge noise spectra across a range of surface lengths, distances, and subsonic jet velocities (which apply during airport operations in both subsonic and supersonic aircraft). A common and simple geometry, a flat plate and a round convergent nozzle, has been used to create general jet-surface configuration upon which an empirical model can be developed, modified, and augmented to provide a first-order approximation applicable to many common aircraft configurations. The jet-surface interaction noise is separated from the jet mixing noise and the surface shielding (or reflecting) effects to model one component of the larger jet-surface interaction problem. This separation is important because it provides a basic building block that can be expanded independent from other considerations; for example, the trailing edge noise may be a function of nozzle aspect ratio ${ }^{1}$ but the noise shielding might only depend on the surface length and span so the a modification factor for aspect ratio can be applied to the jet-surface interaction noise model while the noise shielding model remains unchanged. Once extracted, a series of mathematical functions are fitted onto the far-field jet-surface interaction noise data to give representation of the complete spectra at for any surface length and distance within the limits of the original database. The result is consistent with many system level tools that rely on their ability to quickly run thousands of cases in order to determine a range of the best possible outcomes for more detailed study with actual geometries and jet exit conditions.

\section{Test Setup, Data Acquisition and Processing}

Jet-surface interaction tests were conducted using the Small Hot Jet Acoustic Rig (SHJAR) located in the Aero-Acoustic Propulsion Laboratory (AAPL) at the NASA Glenn Research Center (GRC). The SHJAR is capable of supplying air at flow rates up to $2.7 \mathrm{~kg} / \mathrm{s}$ to a single-steam nozzle. A hydrogen burning combustor is used to simulate core exhaust at temperatures up to $975^{\circ} \mathrm{K}$. Flow conditioning and a lineof-sight muffler are used to achieve a clean and quiet flow at jet exit Mach numbers down to $M_{a}=0.35$. Additional information on the SHJAR, including performance validation data, can be found in. ${ }^{2,3}$ The AAPL is covered with wedges to provide an anechoic environment at frequencies above $200 \mathrm{~Hz}$. To ensure the correct operating conditions, each operating point, as defined by a Mach number and temperature ratio (Table 1), was entered into the AAPL facility data acquisition system, which monitored all relevant rig temperatures and pressures once per second to compute the difference between the current and specified jet exit conditions (based on an L2-norm). The jet condition was required to be within $0.5 \%$ of the specified value throughout the acquisition for an acoustic point to be accepted. The AAPL data system also acquired and stored the ambient conditions for later reference and data corrections.

The configuration common to all these experiments was formed using a flat plate near an axisymmetric $D_{e}=2$ " nozzle (Figure 1). The surface was fabricated using a single plate around the nozzle and a single trailing edge piece. Inserts were used to extend the surface between $1.3 \leq x_{T E} / D_{e} \leq 10$. Each piece was created using $1 / 2$ " thick aluminum sheet with the trailing edge piece tapered to a $45^{\circ}$ angle. The plate was mounted to an automated traverse and allowed to move between $0.5 \leq h / D_{e} \leq 5$ in the radial direction. A complete list of surface positions used for the empirical model is given in Table 2. Note that far-field noise data were acquired on both the 'shielded' $\left(\phi=0^{\circ}\right)$ and 'reflected' $\left(\phi=180^{\circ}\right)$ sides of the surface as identified in Figure 1.

The far-field noise data were acquired using an array of 24 microphones mounted on an arc (150" radius) centered on the jet exit. The $1 / 4$ " Bruel \& Kjaer microphones (type 4939) were placed at $5^{\circ}$ intervals from approximately $50^{\circ}$ upstream to $165^{\circ}$ downstream of the nozzle exit. One additional microphone was mounted on the opposite side of the jet from the primary arc array at approximately $\theta=115^{\circ}$ during testing with surfaces $x_{T E} / D_{e} \leq 12$. Bruel \& Kjaer Nexus units provided signal conditioning and amplification. Data were digitized by a DataMAX Instrumentation Recorded from R. C. Electronics using $200 \mathrm{kHz}$ sample rate (90 kHz Nyquist filter). Once acquired, the time series data were transformed into the frequency domain using a $2^{14}$ point Kaiser window to achieve a $12.21 \mathrm{~Hz}$ spectral resolution. The background noise, as measured at the beginning of each test day, was then subtracted on a frequency by frequency basis; any data point within $3 d B$ of the background noise was removed from the spectra to minimize contamination to the jet noise database. The data were then corrected for the individual frequency response of each microphone using the current calibration obtained from the manufacturer. Finally, the data were corrected for atmospheric attenuation, scaled to a distance of $100 D_{e}$ assuming spherical spreading of sound, and integrated into 1/12 octave power spectral density bands based on Strouhal frequency scaling. 
"Reflected" Observer

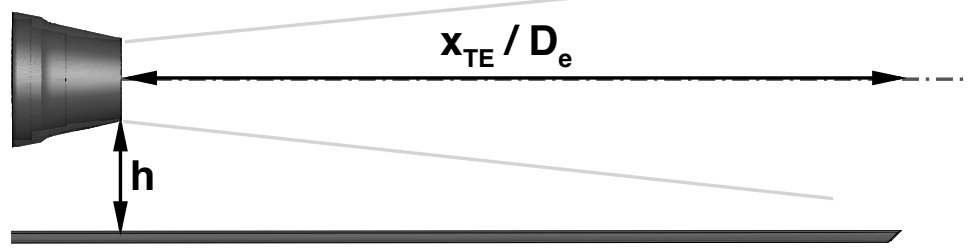

"Shielded" Observer

Figure 1. Schematic showing the configuration tested with the nomenclature used to describe the surface and observed locations.

\begin{tabular}{|c|c|c|c|c|}
\hline Setpoint & $\begin{array}{c}\text { NPR } \\
P_{j} / P_{a}\end{array}$ & $T_{s, j} / T_{a}$ & $\begin{array}{c}M_{a} \\
U_{e} / c_{a}\end{array}$ & $\begin{array}{c}\text { Mass Flow } \\
\mathrm{kg} / \mathrm{s}\end{array}$ \\
\hline 3 & 1.197 & 0.968 & 0.5 & 0.44 \\
\hline 5 & 1.436 & 0.902 & 0.7 & 0.65 \\
\hline 7 & 1.860 & 0.835 & 0.9 & 0.91 \\
\hline
\end{tabular}

Table 1. Subsonic jet exit conditions common to all the jet-surface interaction noise tests.

\begin{tabular}{|c|c|c|c|c|c|c|c|}
\hline$h($ inches $)$ & 0.65 & 1.35 & $\begin{array}{c}x_{T E} / D_{e} \\
2\end{array}$ & 4 & 6 & 8 & 10 \\
\hline 0.0 & $x$ & $x$ & $x$ & $x$ & $x$ & $x$ & $x$ \\
\hline 0.1 & $x$ & $x$ & $x$ & $x$ & $x$ & & \\
\hline 0.2 & $x$ & $x$ & $x$ & $x$ & $x$ & & \\
\hline 0.3 & $x$ & $x$ & $x$ & $x$ & $x$ & & \\
\hline 0.5 & $x$ & $x$ & $x$ & $x$ & $x$ & $x$ & $x$ \\
\hline 0.7 & $x$ & $x$ & $x$ & $x$ & $x$ & & \\
\hline 1.0 & $x$ & $x$ & $x$ & $x$ & $x$ & $x$ & $x$ \\
\hline 1.4 & $x$ & $x$ & $x$ & $x$ & $x$ & & \\
\hline 1.5 & & & & & & $x$ & $x$ \\
\hline 1.9 & $x$ & $x$ & $x$ & $x$ & $x$ & & \\
\hline 2.0 & & & & & & $x$ & $x$ \\
\hline 2.5 & $x$ & $x$ & $x$ & $x$ & $x$ & $x$ & $x$ \\
\hline 3.0 & & & & & & $x$ & $x$ \\
\hline 3.2 & $x$ & $x$ & $x$ & $x$ & $x$ & & \\
\hline 3.5 & & & & & & $x$ & $x$ \\
\hline 4.0 & $x$ & $x$ & $x$ & $x$ & $x$ & $x$ & $x$ \\
\hline 5.0 & $x$ & $x$ & $x$ & $x$ & $x$ & $x$ & $x$ \\
\hline
\end{tabular}

Table 2. Surface lengths $\left(x_{T E} / D_{j}\right)$ and radial positions $(h)$ used to generate the empirical model. 


\section{Model Development}

Jet-surface interaction noise broadly describes an increase in low frequency noise that results when a hard surface is located near turbulent jet. This increase is generally attributed to two source mechanisms: flow 'scrubbing' noise and trailing edge noise. Flow scrubbing noise is formed throughout any region where the high-speed flow passes directly over a hard surface. Trailing edge noise (as the name suggests) originates at the edge of the surface where the flow transitions from wall-bounded to a free-shear layer. Both of these noise sources are dipolar as shown by the mid-1950's theoretical work of Curle. ${ }^{4}$ These theories were later expanded to give scaling as a function of velocity (and surface compliance) and a directivity pattern for the trailing edge noise source. ${ }^{5,6}$ Experimental data has generally supported these theories (e.g. ${ }^{7-9}$ ) and, as a result, they are commonly used. The proposed empirical model leverages these results to greatly simplify development and implementation while improving accuracy.

The empirical model is based on the idea that a spectra can be determined for each measured surface length and standoff at a single flow velocity and observer angle then scaled, using general theories on the behavior of dipole noise sources, to represent other flow conditions and observer angles. These characteristic spectra are determined by a mathematical fit to the available data with care taken to ensure these functions behave in a reasonable manner between the data points (e.g. no diverging polynomials, singularities, etc.). However, the jet-surface interaction noise, must be extracted from total measured noise before a characteristic spectra can be determined.

\section{III.A. Noise Source Decomposition}

The affect of a surface near a jet can be separated into two parts: jet-surface interaction noise source and the noise shielding/reflecting effect. In addition, the measured far-field noise will include the jet mixing noise that is not shielded or reflected jet mixing noise. Figure 2(a) shows an example of these components when the surface is in the jet flow and where jet mixing noise is represented by the isolated (no surface) jet. Even in this case, there is a problem extracting the jet-surface interaction noise by simple subtraction; the extent of the shielding or reflection relative to the jet-surface interaction noise and isolated jet is not fully known. As the surface moves out of the flow, it passes through a region where the flow velocity over the surface is relatively small it is still subject to the hydrodynamic pressure fluctuations present near a high-speed turbulent jet which still create jet-surface interaction noise but at a lower level closer to the jet mixing noise making it much more difficult to extract by simple subtraction (Figure 2(b)). Furthermore, because the jet-surface noise decreases more slowly than the jet mixing noise $\left(U_{e}^{6}\right.$ versus $\left.U_{e}^{8}\right)$, jet-surface noise that is masked by the mixing noise at some frequencies will become exposed as the jet velocity decreases. This signal (jet-surface interaction noise) to noise (jet mixing noise) ratio problem where robust model must extend across a frequency range that will sometimes be hidden by the jet mixing noise; extracting an accurate jet-surface interaction noise spectra from measured noise is critical to building a quality empirical model.

The low signal to noise ratio problem is minimized in two ways: the jet-surface interaction spectra is extracted from data acquired at the lowest jet Mach number $\left(M_{a}=0.5\right)$ to form the characteristic spectra and the relatively high coherence of the surface dipole source. Previous studies have shown that the jetsurface interaction noise produced using a flat surface in similar configurations is dominated by the trailing edge source. ${ }^{9,10}$ Thus, the jet-surface interaction noise should be coherent and $180^{\circ}$ out of phase if measured on both sides of the surface simultaneously. Figure 3 shows representative coherence and phase relationship for a jet-surface configuration as measured simultaneously on opposite sides of the surface. The jet mixing noise, represented by the isolated jet, is highly incoherent between these measurement locations as opposed to the trailing edge noise which is coherent and, as expected of a dipole, $180^{\circ}$ out of phase. Similar coherence data can be extracted for each surface configuration and flow condition. These data can then be used to extract the percentage of coherent (dipole) noise from each measured spectra providing an estimate of the sound energy associated with the jet-surface interaction noise (or mathematically, $P_{d}=P_{t} * \gamma_{x y}^{2}$ where $P_{t}$ and $P_{d}$ are in sound power not $\mathrm{dB}$ ). Figure 4 shows the results of this source decomposition procedure one case with dominant jet-surface interaction noise and one case when the trailing edge noise source is relatively weak. In each case the decomposition is verified by adding the extracted energy to the sound measured from an isolated jet (equivalent Mach number) to reconstruct the original measured jet-surface spectra (note that noise shielding is not included in the reconstruction the spectra at higher frequencies follows the isolated jet rather than the shielded jet). Notice in the second configuration (Figure 4(b)) where the dipole spectra changes slope around $S t_{D e}=0.35$. This is the point where the coherence has devolved to levels similar to 
the isolated jet (i.e. the jet-surface dipole has no energy) which are low but not quite zero. These frequencies need to removed from the jet-surface spectra prior to the mathematical fitting process either by setting a threshold below which the coherence is set to zero or by limiting the frequency range allowed in by the fit procedure or the modeled jet-surface spectra will be far too broad in many cases (using the fit method described below). However, comparing back to Figure 4(a), this point will be different for each surface position so the coherence method is generally preferred.

The data available at most surface positions could be decomposed using the coherence method with a microphone on each side of the surface. However, the longest two surfaces $\left(x_{T E} / D_{e} \geq 8\right.$ in Table 2) were only tested in one entry and did not include this opposite side microphone. These lengths were considered important to the model because their inclusion would ensure the model includes at least some surface that extend downstream past the end of the jet's potential core for most subsonic exit conditions. The jet-surface noise was extracted from the spectra at these configurations using the coherence between two microphones on the same side but at different polar angles $\left(\theta=90^{\circ}\right.$ and $\left.\theta=120^{\circ}\right)$. The jet mixing noise is relatively incoherent across these angles while the dipole coherence remains strong. Source separation based on these polar angles was generally successful but does introduce more uncertainty at these locations compared to the other points (particularly when the surface is away from the flow).

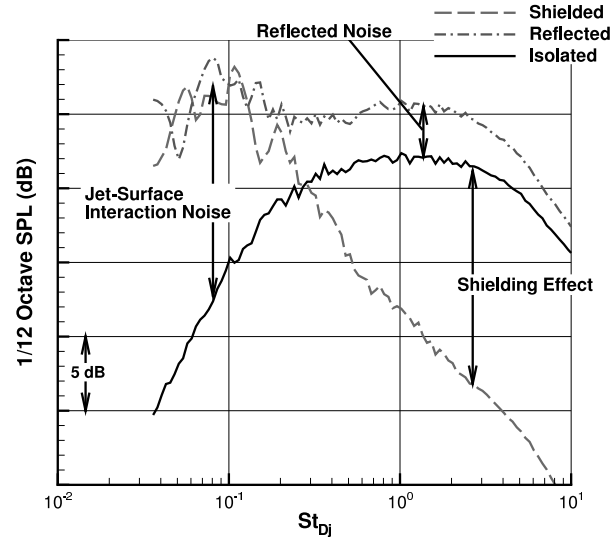

(a) $x_{T E} / D_{e}=15, h=1$

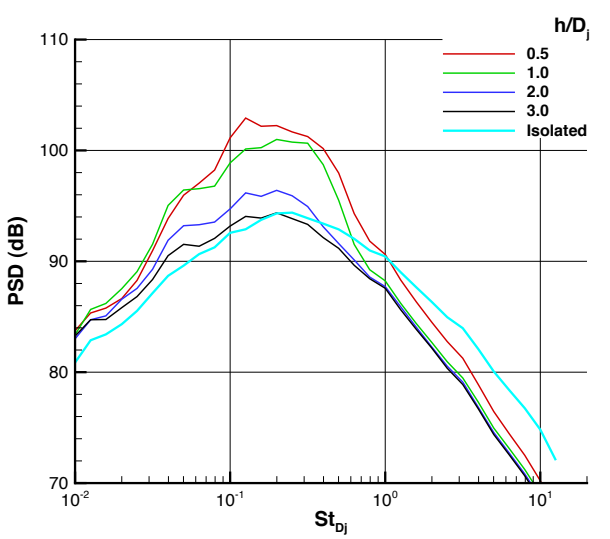

(b) $x_{T E} / D_{e}=6$

Figure 2. Jet-surface interaction noise, jet mixing noise (Isolated), noise shielding, and reflected noise labeled for a typical jet-surface arrangement where the flow is directly over the trailing edge (left) and changes in the jet-surface noise as the surface moves away from the jet (right).

\section{III.B. Jet-Surface Noise Model}

The jet-surface interaction noise spectra, extracted from the total measured noise, can be modeled in many different ways which all have the same goal: to describe the behavior within some parameter space in the simplest possible form given the accuracy requirements for the given problem. One common method is to define a series of basis functions and use a singular value decomposition fit to determine coefficients for each function at each frequency and/or angle. This may result in a high number of coefficients but computers are remarkably good at dealing with these. This was the first method tried on the jet-surface interaction data but several problems were encountered. The most significant of these was that the relatively low signal to noise ratio created a situation where not all configurations yielded valid data at each frequency and, as a result, the model was biased toward the configurations with higher jet-surface interaction noise. And while it is possible to extrapolate based on the available data to restore some of the missing data, this process introduces its own modeling assumptions. It was, therefore, decided to use the assumptions at this point to apply a different modeling method.

The jet surface interaction noise spectra depends on several variables: surface length, surface distance, jet velocity (or Mach number), jet temperature, and observer angle. Of these variables, theory and experimental evidence gives guidance to changes in jet velocity and observer angle and jet temperature is not yet considered in the current data set. That leaves the surface length and distance as the two remaining fit variables. If a model provided a characteristic spectra within the range of available surface positions then that spectra 


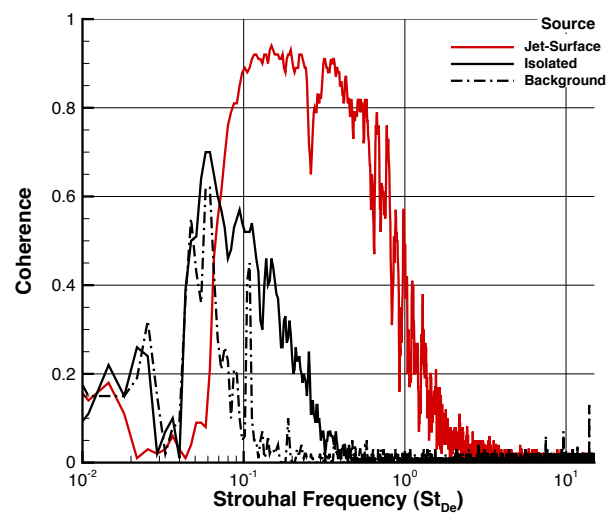

(a) Coherence

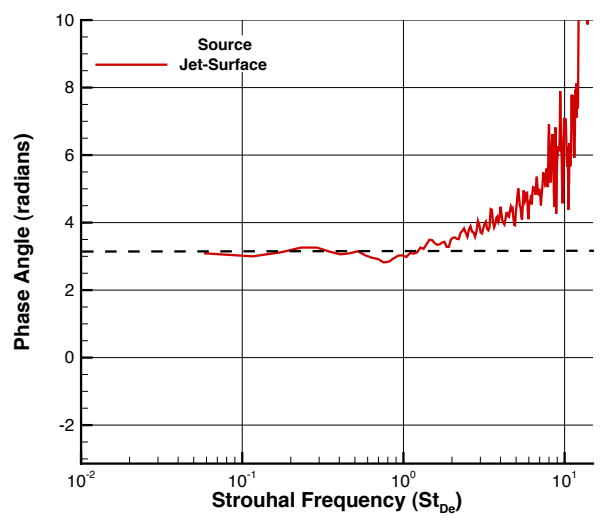

(b) Phase

Figure 3. Coherence of a jet with a surface relative to an isolated jet and the AAPL background noise (left) and the phase relationship measured between two microphones on opposite sides of the jet-surface configuration (right). The horizontal dashed line locates the $180^{\circ}(\pi)$ phase angle.

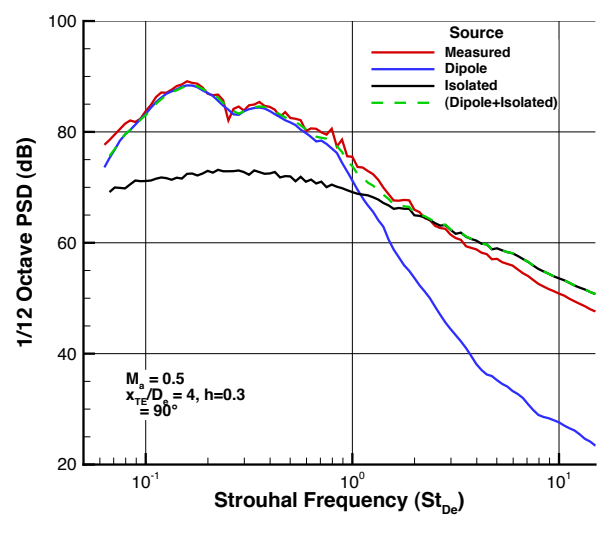

(a) $x_{T E} / D_{e}=4, h=0.3$

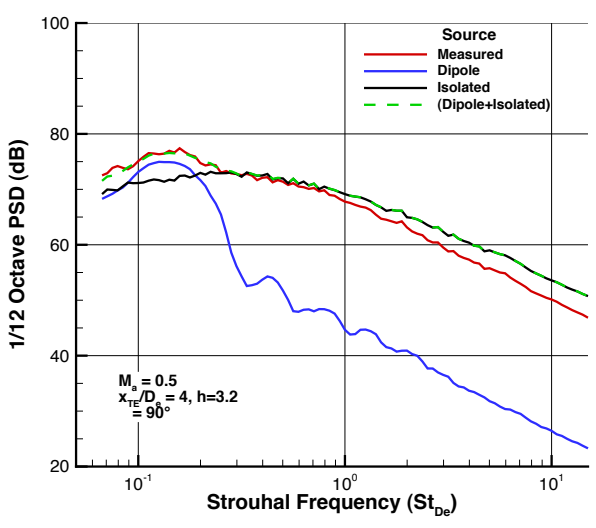

(b) $x_{T E} / D_{e}=4, h=3.2$

Figure 4. Source decomposition and reconstruction using the coherence based method at two surface positions and a jet Mach number $M_{a}=0.5$. Note that the reconstructed spectra collapse to the isolated jet spectra rather than the measured spectra because the noise shielding effect is not included in the model. 
could be scaled through the range of the other parameters. Looking at the jet-surface interaction spectra (e.g. Figure 4), a parabolic model was selected for the characteristic spectra giving the model:

$$
P_{d}=A+B \log _{10}\left(f / F_{\text {peak }}\right)^{2}
$$

where $A$ gives the peak amplitude, $B$ describes the spectral width, and $F_{\text {peak }}$ locates the spectral peak. The advantage of this model is that the three coefficients $\left(A, B\right.$, and $\left.F_{\text {peak }}\right)$ can be determined using a minimum number of frequencies and then extended to cover the entire frequency space as desired in effect extrapolating based on the "best" data available. Furthermore, the experimental data show that the jet-surface interaction noise spectra generally has a single peak and decreases to a level significantly below the jet mixing noise before changing slope (a point which is likely to be the measurement noise floor as opposed to a physical characteristic of the jet-surface interaction noise). So allowing a parabolic model to decrease to some very low levels at high and very low frequencies will not affect the overall predicted spectra.

A least-squares fit was used to identify the model coefficients for each individual spectra. Although the model equation is not linear with respect to the peak frequency term $\left(F_{\text {peak }}\right)$, it is linear in the amplitude $(A)$ and spectral width terms $(B)$ so a range of peak frequencies $\left(0.01 \leq S t_{D e} \leq 5\right)$ was used. The leastsquares fit was then repeated at increments of 0.025 within this range to determine $A$ and $B$ as well as the fit residual. The value of $F_{\text {peak }}, A$, and $B$ that yielded the lowest fit residual were then stored as the fit parameters for that jet Mach number, surface length, and surface distance. This process allows a fairly straightforward fit method to get the model parameters at each configuration but leads to a situation where the accuracy of the coefficients is dependent on the number of frequencies available to the fit but (1) the fits with more uncertainty are likely to have fewer points exerting less influence on the spectra particularly at the frequencies where data is missing and (2) the parabolic model equation will force a decreasing amplitude away from the peak frequency as observed in the coherence plots (e.g. Figure ??) as long as $B<0$ (this should be checked as $B$ may become positive when the velocity over the trailing edge is low because of a long surface or a low $U_{e}$ ). This fit would provide the extrapolation to the missing frequencies and, therefore, could be put back into another fit method (like the previously mentioned frequency by frequency singular value decomposition for example). Alternatively, if all these fit spectra are considered together, Equation (1) can be written as:

$$
P_{d}\left(x_{T E} / D_{e}, h\right)=A\left(x_{T E} / D_{e}, h\right)+B\left(x_{T E} / D_{e}, h\right) \log _{10}\left[f / F_{\text {peak }}\left(x_{T E} / D_{e}, h\right)\right]^{2}
$$

In this form each coefficient is considered its own function of surface length and position. Each coefficient can be modeled or fit to the output data from the previous least-squares fit and, when combined, give a characteristic spectra for each surface position in the domain. Once determined for each surface length and position, the characteristic spectra can be adjusted for jet velocity and observer angle using known scaling laws for dipole sources. Expanded $P_{d}$ to include these variables would give the equation:

$$
P_{d}\left(x_{T E} / D_{e}, h, M_{a}, \theta\right)=\left[A+B \log _{10}\left(f / F_{\text {peak }}\right)^{2}+60 \log _{10}\left(M_{a} / M_{f i t}\right)\right]\left[1-\cos ^{2}(\theta)\right]
$$

where $M_{f i t}$ is the Mach number used to generate the $A, B$, and $F_{\text {peak }}$ and $\theta$ is polar angle. Furthermore, future modifications and additions may be applied to the model by introducing new terms to the coefficient equations; for example, jet exit temperature may be included by introducing a new function to the amplitude coefficient $(A)$ so that it becomes $A\left(x_{T E} / D_{e}, h\right) * T_{d}\left(T_{t r}\right)$ (where $T_{d}$ is a function giving the amplitude change of the dipole noise as a function of total temperature ratio, $\left.T_{t r}\right)$. Similar modifications could be applied to the other coefficients ( $B$ and/or Fpeak). Other parameters, such as nozzle aspect ratio, might also be included in this way.

\section{III.B.1. Defining $A\left(x_{T E} / D_{e}, h\right)$}

The model given by Equation 2 requires three coefficients that are each a function of surface length and position. The first of these, $A$, gives the characteristic model spectra its peak amplitude. To develop a mathematical fit, the values of $A$ determined using the least-squares parabolic fit are plotted as a function of $h$ for each measured $x_{T E} / D_{e}$ given in Table 2. These plots are shown in Figure 5. The initial expectation based on previous test data was that the peak amplitude would decrease logarithmically as $h$ increased and, in fact, a logarithmic function could be used if the model began in the $0.5 \leq h \leq 1$ region. However, as the surface moves closer to the jet $A$ peak in the $0.3 \leq h \leq 0.5$ range and then decreases again as $h$ goes to zero. 
One hypothesis for this behavior is that a surface very near the jet will suppress the turbulence over the trailing edge (possibly by limiting entrainment air) which reduces the peak trailing edge noise. In this case, the peak amplitude would occur at a position just off the nozzle and then decrease as the surface proceeds out of the flow. Therefore, a cubic polynomial function is used to fit the $A$ coefficient as a function of $h$ at each fixed value of $x_{T E}$ in an effort to capture this behavior. Note that the fit is quite poor (based on $R^{2}$ ) for the shortest surfaces because the surface moves out of the flow very quickly. However, the values of $A$ when the surface is at $x_{T E} / D_{e}=0.65$ are quite close together (notice the scale in Figure $5(\mathrm{a})$ ) so the impact of the difference on the overall spectra is relatively small while the rapid fall off with distance at $x_{T E} / D_{e}=1.35$ leads to maximum $2 d B$ difference at the peak. Also, as the surface gets longer the maximum value of $A$ occurs when the surface in slightly farther away from the jet. Finally, both the values from Equation (2) and the corresponding polynomial fit are not as smooth and predictable as when the surface is shorter because (1) there are fewer data points at these surface lengths to fit and (2) the jet-surface interaction noise was extracted using the coherence at two polar angles rather than the coherence on opposite sides of the surface.

The individual cubic polynomial fits shown in Figure 5 can provide the peak amplitude coefficient $(A)$ to Equation (2) for the discrete values of $x_{T E} / D_{e}$ measured. The desired model, however, needs to provide an estimated jet-surface interaction noise spectra for any value of $x_{T E} / D_{e}$. Thus, the coefficients of individual polynomial fits are considered as data points for another fit across all surface lengths so that each coefficient $\left(A_{x^{n}}\right)$ becomes is a function of $x_{T E} / D_{e}$. Mathematically, the $A$ coefficient to Equation (2) is now written as:

$$
A\left(x_{T E} / D_{e}, h\right)=A_{x^{3}}\left(x_{T E} / D_{e}\right) h^{3}+A_{x^{2}}\left(x_{T E} / D_{e}\right) h^{2}+A_{x^{1}}\left(x_{T E} / D_{e}\right) h^{1}+A_{x^{0}}\left(x_{T E} / D_{e}\right)
$$

Separating the coefficients so that a set based on one variable provides the second set allows the model to cover a 2-dimensional parameter space while using only 1-dimensional fit methods. In exchange, each coefficient $\left(A_{x^{3}}, A_{x^{2}}\right.$, etc. $)$ requires a different fit creating another separate set of coefficients.

The behavior of $A$ as a function of $h$ was reasonably described by a third-order polynomial at the individual surface lengths $\left(x_{T E} / D_{e}\right)$ where data were acquired. The coefficients of that polynomial, however, are not as easily described by simple functions of $x_{T E} / D_{e}$. Figure 6 shows the fit results to the the $A_{x^{n}}$ coefficients in Equation 4. A general polynomial equation was used for each fit with a few restrictions applied. First, no polynomials above $3^{\text {rd }}$ order were allowed. Higher order polynomials were found to fit the data points but these functions were unpredictable between the data points. Second, regions with sharp changes (e.g. $x_{T E} / D_{e} \approx 2$ in Figure $6(\mathrm{~b})$ ) or where fourth order polynomials (or higher) were needed were sub-divided into multiple regions with different functions/coefficients to best represent the nearby data. Each division increases the complexity of the overall model but allows for a more predictable result between data points. Finally, the trend the lower and upper limits of the data was considered in an effort to avoid any non-physical divergence immediately outside of the fit range (note that this was more successful on the lower end of surface lengths). Figure 6 shows the fit results compared to the data points for the 4 coefficients in Equation 4 (regions, functions, and coefficients can be found in Appendix A). It is important to remember here that the results of these fits proved the coefficients to another equation and, therefore, are two steps removed from the actual physics (with the exception of $A_{x^{0}}$, Figure $6(\mathrm{~d}$ ), which represents the behavior of $\left.A\left(x_{T E} / D_{e}, h=0\right)\right)$. The fit data and results for the $A_{x^{n}}$ coefficients is shown to illustrate the process of fitting equations to the coefficients of another equation to get a usable model. It is how these coefficients combine in Equation 4 that provides the characteristic spectra with its peak amplitude.

The coefficients $A_{x^{n}}\left(x_{T E} / D_{e}\right)$ are obtained through a fit to the coefficients of a third-order polynomial (in $h$, Figure 5) across all values of surface length $\left(x_{T E} / D_{e}\right)$. These coefficients are used with Equation 4 to get the peak amplitude term $\left(A\left(x_{T E} / D_{e}, h\right)\right)$ in the jet-surface interaction noise model (Equation 2). Figure 7 shows how the peak amplitude varies in the jet-surface interaction noise model for six surface distances $(h)$ and all surface length within the limits of the original data (Table 2). When the surface is on the nozzle lipline $(h=0)$ there is flow across the surface trailing edge for any length of surface. As a result, the amplitude of the jet-surface interaction noise varies smoothly increases as the surface length increases (possibly) because the boundary layer on the surface has more time to develop and, thus, the turbulence intensity is greater at the trailing edge (and, presumably, if a surface were long enough the amplitude would decrease because the flow passing over the trailing edge would not have enough energy to create as much sound). When the surface is at $h=1$, the shortest surfaces are no longer subject to the flow directly as reflected in the lower amplitudes. A transition occurs between $2 \leq x_{T E} / D_{e} \leq 5$ where the flow first strikes the surface and develops a turbulent boundary layer. When this occurs, the peak amplitude matches the 
level at $A\left(x_{T E} / D_{e}, h=1\right)$. Beyond $h=1$ the surface appears to be in a lower velocity/turbulence region as it passes through the hydrodynamic field of the jet $(2 \leq h \leq 3)$, dropping in amplitude as $h$ increases. Finally, the $A\left(x_{T E} / D_{e}, h\right)$ settles to about the same level/profile which may be indication that the jet-surface noise decomposition has reached the signal to noise ratio limit. In this region $(4 \leq h \leq 5)$ the surface is far enough away to significantly weaken the trailing edge dipole source making the surface more a shield for the jet mixing noise than a noise source itself and the peak value collapses to some background noise level. That it does not continue to decrease does not generally appear to be an issue because the jet mixing noise will be sufficiently above this level at least for jet Mach numbers greater than $M_{a}=0.5$.

Finally, a note about the behavior of $A\left(x_{T E} / D_{e}, h\right)$ in the $8 \leq x T E / D_{e} \leq 10$ should be added here as the character of the curves changes significantly in this region. The source data in this region included fewer $h$ and the source decomposition of these data points used two polar angles rather than to points on opposite sides of the surface. So while the coefficient fit in this region showed a similar accuracy to other areas (often due to a separate set of fit equations, see Figure 6), these issues with the source data permeate the model and appear in the output. Therefore, improving the data quality in this region should improve (and possibly simplify) the model.

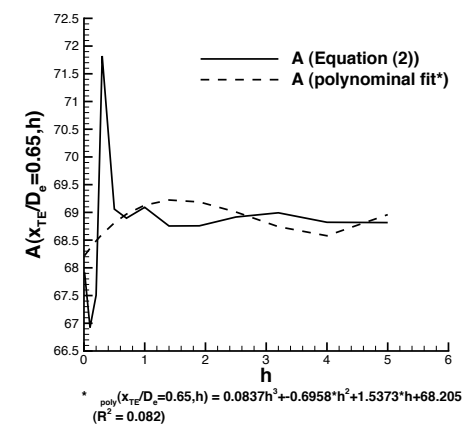

(a) $x_{T E} / D_{e}=0.65$

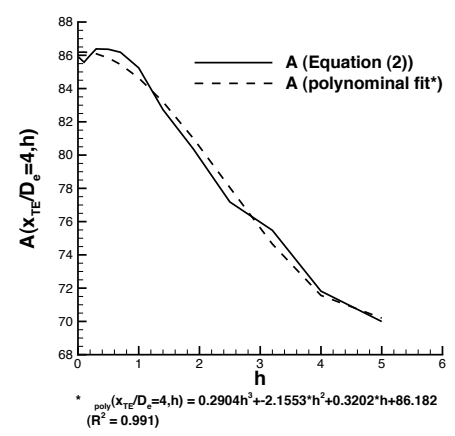

(d) $x_{T E} / D_{e}=4.0$

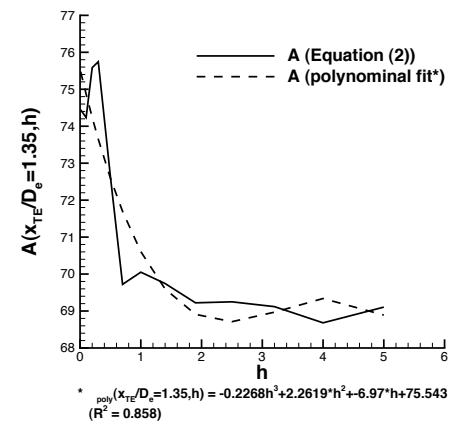

(b) $x_{T E} / D_{e}=1.35$

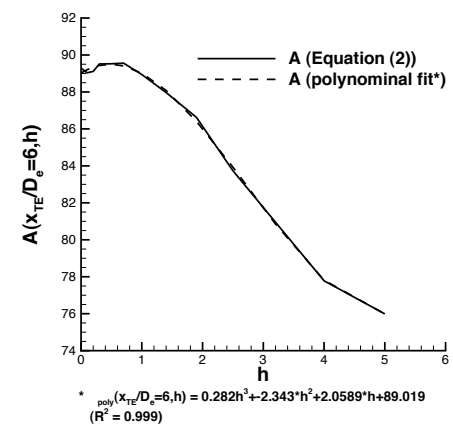

(e) $x_{T E} / D_{e}=6.0$

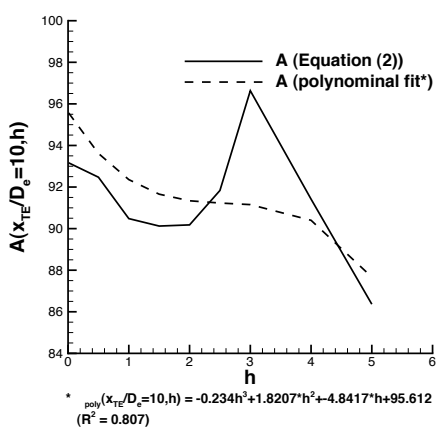

(g) $x_{T E} / D_{e}=10.0$

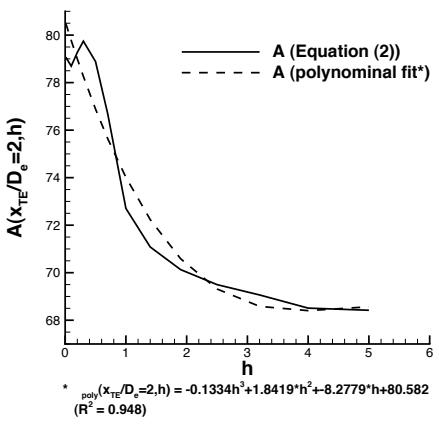

(c) $x_{T E} / D_{e}=2.0$

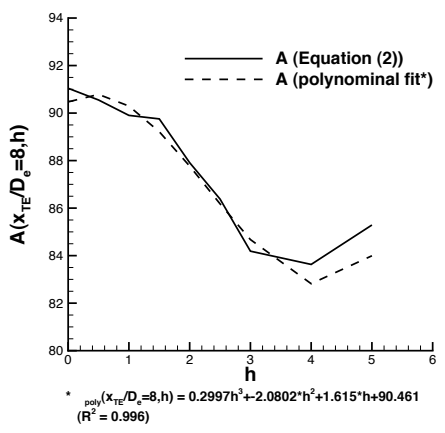

(f) $x_{T E} / D_{e}=8.0$

Figure 5. Values of $A\left(x_{T E} / D_{e}, h\right)$ determined by the fit process using Equation (1) and the cubic polynomial fit to $A\left(x_{T E} / D_{e}, h\right)$ determined at each $x_{T E} / D_{e}$. Note the scale change on the $y$-axis of each plot. 


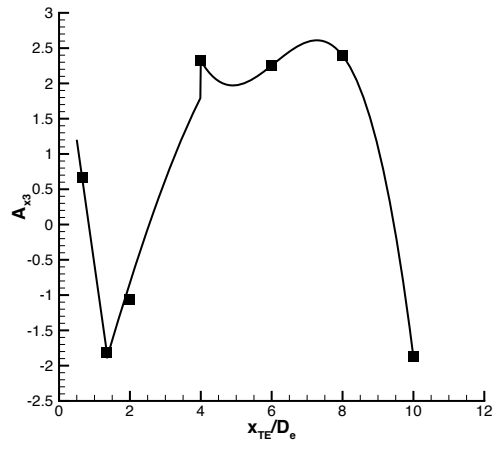

(a) $A_{x^{3}}$

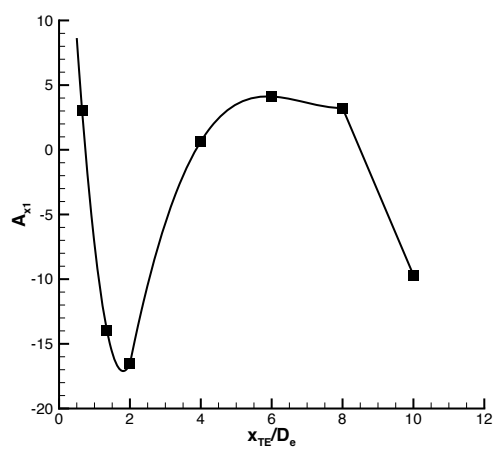

(c) $A_{x^{1}}$

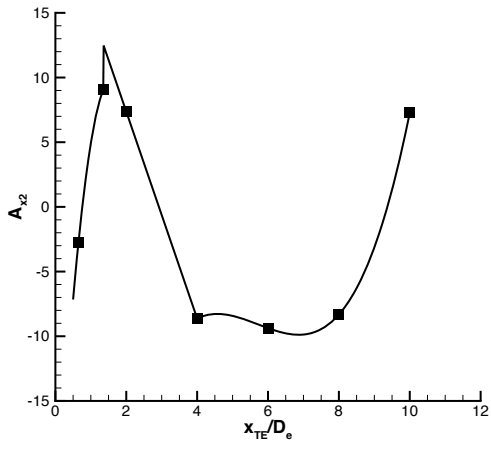

(b) $A_{x^{2}}$

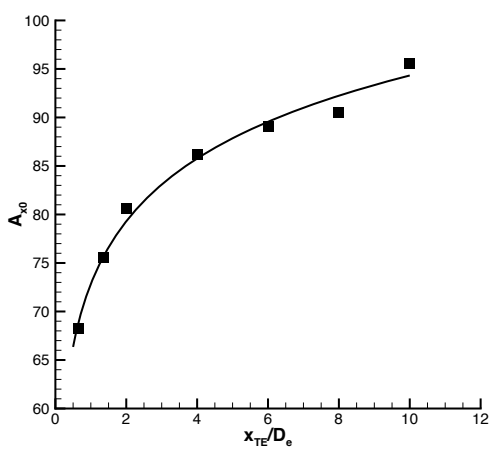

(d) $A_{x^{0}}$

Figure 6. Fit results (line) with input data points (squares) for each coefficient to Equation 4 . Fit equations, coefficients, and ranges of applicability are given in Appendix A.

\section{III.B.2. Defining $B\left(x_{T E} / D_{e}, h\right)$}

The characteristic spectra for the jet-surface interaction model is defined by a parabolic equation peak amplitude, spectra width, and peak frequency terms. The peak amplitude was defined by an empirical fit to the available data in Section III.B.1. The spectral width $\left(B\left(x_{T E} / D_{e}, h\right)\right)$ is determined using the same general procedure. First, the values of $B\left(x_{T E} / D_{e}, h\right)$ determined by a parabolic fit to the jet-surface interaction noise spectra as measured at each surface position. A third-order polynomial function of $h$ is then fit to these values at each surface length (e.g. $B\left(x_{T E} / D_{e}=0.65, h\right), B\left(x_{T E} / D_{e}=1.35, h\right), B\left(x_{T E} / D_{e}=2.0, h\right)$, etc.). The term by term coefficients of these polynomials are then used as source data to fit another series of first- to third-order polynomial functions of $x_{T E} / D_{e}$. The coefficient $B\left(x_{T E} / D_{e}, h\right)$ is then obtained by working back through this process; $x_{T E} / D_{e}$ is used to find the coefficients to the cubic polynomial function of $h$ which is in turn used to calculate the spectral width. Mathematically, the equation for $B\left(x_{T E} / D_{e}, h\right)$ written:

$$
B\left(x_{T E} / D_{e}, h\right)=B_{x^{3}}\left(x_{T E} / D_{e}\right) h^{3}+B_{x^{2}}\left(x_{T E} / D_{e}\right) h^{2}+B_{x^{1}}\left(x_{T E} / D_{e}\right) h^{1}+B_{x^{0}}\left(x_{T E} / D_{e}\right)
$$

which has the same form used in Equation 4 for $A\left(x_{T E} / D_{e}, h\right)$. The difference arises in the fit equations (with applicable regions) and coefficients used to determine the values $B_{x^{3}}, B_{x^{2}}, B_{x^{1}}$, and $B_{x^{0}}$. The process itself, however, is identical to that used to determine $A\left(x_{T E} / D_{e}, h\right)$ in Section III.B.1 and so will not be repeated here in such detail. The results for several surface distances $(h)$ are shown in Figure 8 while the fit coefficients and equations can be found in Appendix B. Note that the $B$ parameter required more sub-regions than $A$ primarily to keep the polynomial fit equations from giving positive values. Still there are a few instances where positive values can occur (e.g extrapolating to $x_{T E} / D_{e}<0.65, h=0$ or at $x_{T E} / D_{e}>6, h=5$ ) and, therefore, a limiter is recommended to keep $B \leq-10$. 


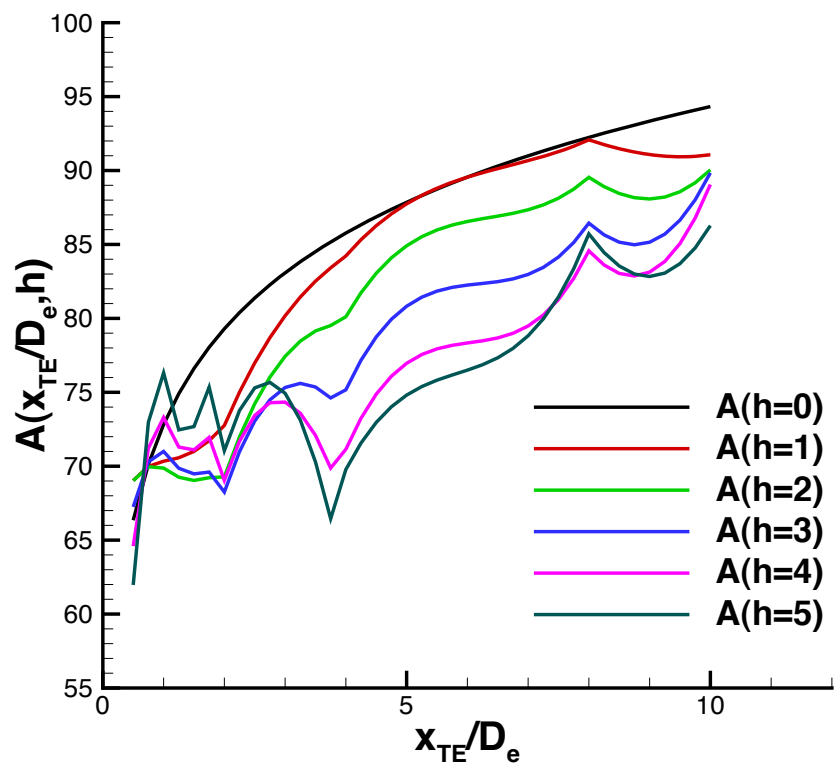

Figure 7. $A\left(x_{T E} / D_{e}, h\right)$ obtained using the fit results shown in Figure 6 for 6 values of $h$.

The parabolic model requires that $B\left(x_{T E} / D_{e}, h\right)$ remain less than zero to maintain its convex shape. Smaller values of $B\left(x_{T E} / D_{e}, h\right)$ will give the jet-surface interaction noise spectra a narrow peak while values approaching zero gives a spectra covering a broad range of frequencies. However, the mechanisms behind the trends in spectra width is not easily identified. Jet mixing noise theory holds that high frequency noise originates from the small-scale turbulence in the thin shear layer near the jet while lower frequencies are generated by the large-scale turbulence structures farther downstream in the fully developed jet. The combination of these source (with the intermediate turbulence scales in between) gives the jet mixing noise spectra its characteristic broadband shape. If this relationship between turbulence scale and noise were applied to the jet-surface interaction noise spectra, then shorter surfaces should have a relatively narrow peak (smaller $B$ ) because of the relatively few small range of turbulence scales in the flow at the surface trailing edge. Similarly, as the surface moves away from the jet ( $h$ increasing) the spectral width should decrease because only the large-scale turbulent structures have sufficient energy to exert influence outside of the jet column. Figure 8 shows the spectral width parameter $(B)$ as a function of surface length. When the surface is on the nozzle lipline $(h=0)$ the jet-surface interaction noise spectra has a near constant width for $x_{T E} / D_{e}>4$ (which may correspond to the length required to develop a fully turbulent boundary layer on the surface). A similar trend, although at a lower overall level, is found at $h=1$ but for $x_{T E} / D_{e}>6$ presumably because a longer surface is need for the jet to spread to the surface then develop a boundary layer. This trend continues out the $h \approx 3$. The spectral width for shorter surfaces or surfaces farther away is generally not easily explained in fluid dynamic terms (e.g. the low point at $x_{T E} / D_{e}=5$ or the broadening at $h=5$ ). Rather it is likely that the fit in these regions is heavily influenced by the spectral decomposition and the related signal to noise ratio issues discussed in Section III.A. Fortunately the spectral width plays a secondary role to the peak amplitude in these regions; as long as the peak amplitude decreases as predicted, the extra wide parts of the predicted spectra will be masked by the jet mixing noise.

\section{III.B.3. Defining $F_{\text {peak }}\left(x_{T E} / D_{e}, h\right)$}

The final parameter to model the jet-surface interaction noise spectra is the peak frequency $\left(F_{p} e a k\right)$ in the parabolic model. The procedure used to obtain a usable fit for $F_{p e a k}\left(x_{T E} / D_{e}, h\right)$ is identical to that used to determine values for $A$ and $B$ in the previous two sections (so a detailed description will not be repeated here) with the exception that a second-order polynomial was suitable to describe $F_{\text {peak }}$ as a function of $h$. Thus, the equation for $F_{\text {peak }}\left(x_{T E} / D_{e}, h\right)$ is: 


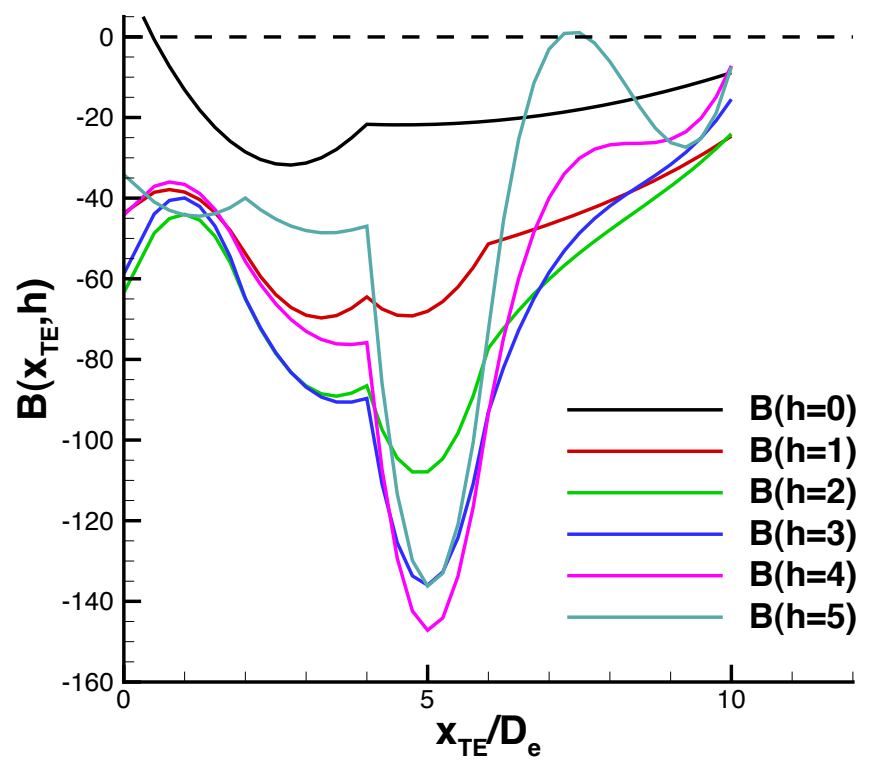

Figure 8. $B\left(x_{T E} / D_{e}, h\right)$ obtained using the fit results in Appendix B for 6 values of $h$. A dashed horizontal line is shown at $B\left(x_{T E} / D_{e}, h\right)=0$ as the convex shape of the parabolic jet-surface noise model requires $B\left(x_{T E} / D_{e}, h\right)<0$.

$$
F_{\text {peak }}\left(x_{T E} / D_{e}, h\right)=F_{x^{2}}\left(x_{T E} / D_{e}\right) h^{2}+F_{x^{1}}\left(x_{T E} / D_{e}\right) h^{1}+F_{x^{0}}\left(x_{T E} / D_{e}\right)
$$

where the coefficients $F_{x^{n}}$ are functions of $x_{T E} / D_{e}$ and provided by the fit equations and coefficients shown in Appendix C. Figure 9 shows how the peak frequency of the jet-surface interaction noise spectra predicted by the model changes as a function of $x_{T E} / D_{e}$ at several surface distances $(h)$. Like the spectral width, the peak frequency could be related to the turbulence in the flow at the trailing edge. In this hypothesis, surfaces close to the jet (e.g. $h=0$ ) would have a higher peak frequency than found in spectra measured with surfaces farther away which would be more affected by the large-scale turbulence. As Figure 9 shows this trend generally holds for the peak frequencies plotted. The second part of this hypothesis, concerning surface length, would suggest that the peak frequency would decrease as the surface length increases because the dominate flow structures would be larger. Again, Figure 9 shows that after an initial increase (presumably while the boundary layer develops on the surface), this trend at all surface distances. Note that this is not sufficient evidence to prove this flow to noise link but to point out interesting trends that may be observed by modeling the data in this way and discuss how those may relate to the fluid dynamics for future theory, experiments, or modeling efforts.

The peak frequency term of the characteristic spectra model was the easiest of the three variables to fit. Each term required only two regions and most of these were sufficiently described by quadratic polynomials. However, the fitted peak frequency shown in Figure 9 shows a couple of issues related to the fit. First, there is a rapid jump around $x_{T E} / D_{e}=2$ which develops when the surface is around $h=4$. While not ideal, this was not considered a major issue because the jet-surface interaction noise is generally low relative to the jet mixing noise with surfaces that are short and far from the jet so that the affect on the reconstructed spectra is small. Second, the peak frequency trends to negative values for longer surfaces $\left(x_{T E} / D_{e}>8\right)$ that are far from the jet $(h>4)$. The peak frequency identified by the original parabolic fit to Equation 2 for the points in this region was the minimum allowed in the fit routine. Therefore, a limiter was placed on $F_{\text {peak }}$ so that the predicted value will not go below the range specified in the original fit (note that the $A$ and $B$ values fit correspond this minimum peak frequency so allowing it to go outside of the original range may have unintended consequences). Finally, the peak frequency is somewhat unpredictable when extrapolated to surfaces shorter $x_{T E} / D_{j}=0.65$. Cutting the model off at $x_{T E} / D_{e}=0.5$ (as in $A$ and $B$ ) limits the spread in this region so it may or may not be a problem; there is no validation data in this region so this behavior should be noted at this point for future evaluation. 


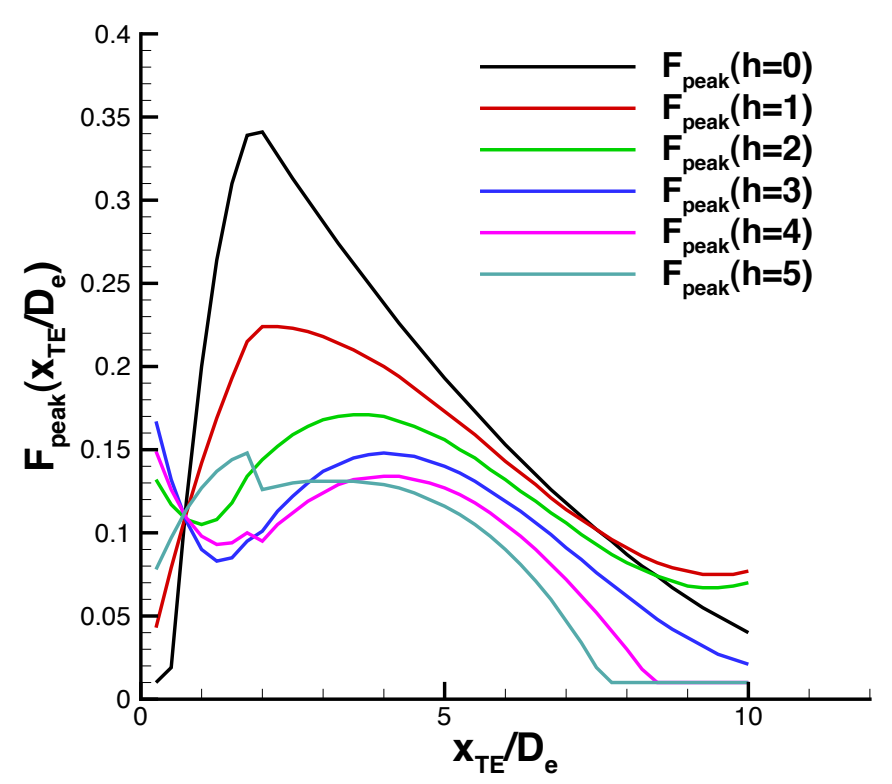

Figure 9. $F_{\text {peak }}\left(x_{T E} / D_{e}, h\right)$ obtained using the fit results in Appendix $\mathbf{C}$ for 6 values of $h$. Note the limiter that engages for longer surfaces at the farthest two surface positions to ensure a positive frequency.

\section{Model Evaluation and Performance}

An empirical model has been developed based on the idea that a characteristic jet-surface interaction noise spectra can be generated across a range of surface lengths and positions using a parabolic equation with coefficients selected to fit experimental data at one jet velocity and one observer angle. The characteristic spectra, according to the model, can then be scaled to other jet Mach numbers and/or observed angles using the theoretical rules for a dipole noise source. Furthermore, the scaled jet-surface interaction noise spectra can be added to a modeled jet mixing noise spectra to recreate the complete noise source spectra (note that shielding or reflection of the jet mixing noise is not included in the reconstruction). The jet-surface interaction noise model is evaluated on these three performance metrics: (1) matching the characteristic spectra to the experimental data, (2) scaling the characteristic spectra to account for changes in jet exit velocity, and (3) scaling the characteristic spectra to predict the noise at other observer angles. An empirical jet mixing noise prediction model ${ }^{11,12}$ is combined with the jet-surface interaction noise model for these comparisons.

\section{IV.A. Characteristic Spectra}

The first test of any empirical model is how well it reproduces the source data used to develop it. The volume of data, however, makes it difficult to present comparisons for every data point. Therefore, the model is compared back to the source data to show a comparison in trends as $h$ increases and a comparison of trend as $x_{T E} / D_{e}$ increases with special attention to areas where discrepancies appear. Figure 10(f) shows the decrease in jet-surface interaction noise as a mid-length $x_{T E} / D_{e}=6$ surface moves from $h=0$ to $h=5$. The agreement is good with the model capturing the decrease in peak amplitude and frequency as $h$ increases. The noise from the equivalent isolated (no surface) jet is also included in Figure 10(f); the spectral width of the jet-surface interaction noise is shown by the frequency where the jet-surface interaction noise cross the isolated jet (above this frequency the model returns the isolated jet spectra because a noise shielding model has not been included). The model accurately predicts the cross over frequency and spectral width across the full range of $h$.

One issue not addressed by Figure 10(f) but worthy of special attention is the spectral changes between 
$h=0$ and $h=0.5$ where the data motivated the use of a cubic polynomial to fit the peak amplitude coefficient in Section III.B.1 rather than the logarithmic relationship initially expected. Figure 11 shows predicted and measured spectra for surface lengths $x_{T E} / D_{e}=2$ and $x_{T E} / D_{e}=4$ for each measured $0 \leq h \leq 0.5$. First, at $x_{T E} / D_{e}=4$ (Figure 11(b)), where the amplitude coefficient $(A)$ as a function of $h$ followed a more parabolic trend that was almost flat around the peak between $0 \leq h \leq 0.5$ (Figure $5(\mathrm{~d})$ ), the model produces a reconstructed spectra with a peak at $F_{\text {peak }} \approx 0.2$ that does not vary significantly in amplitude. The peak in the measured spectra, however, occurs as a spectral hump peaking around $S t_{D e}=0.17$ and decreasing as $h$ increases. This does not, however, appear to be a problem fitting the peak amplitude but rather a limitation of the parabolic model used to define the characteristic spectra; the actual peak of the measured data is an extra hump on top of the underlying parabolic jet-surface noise interaction spectra. Similarly, the measured spectra contains several tonal spikes (particularly for $h=0.2-0.3$ ) that can not be captured by a strictly parabolic model but, along with the extraneous peak, influence the spectra width resulting in the spread across the predicted noise between $0.4 \leq S t_{D e} \leq 1.0$ where the jet mixing noise supports the unshielded spectral reconstruction. At $x_{T E} / D_{e}=2$ (Figure 11(b)) the jet-surface interaction peak is not as far above the underlying parabolic form but the mid-frequency tonal content is significantly stronger and occurs to some extent for all $0 \leq h \leq 0.5$. These resonances have been documented in rectangular nozzle/surface configurations and data indicates that have fluid dynamic origins (i.e. structural or plate vibrations). ${ }^{1,13}$ Furthermore, these resonances appear through a relatively small combination of flow velocity and surface length/distance making them difficult to capture in any predictive model. As stated above, the current parabolic jet-surface interaction noise model is functionally incapable of warping to these tonal peaks but these could be modeled separately and implemented as a addition to the parabolic characteristic spectra ${ }^{a}$ As a side note, attempts to model these spectral features on a frequency by frequency basis spanning surface positions and flow velocities required functions that made the model somewhat unstable at other positions.. Thus, this tonal content is less of a concern than the model under-predicting the lower frequency peak at these surface positions because of the underlying parabolic spectra assumption.

The influence of these tones on the predicted jet-surface interaction noise spectra is not limited to changes in surface distance. Figure 12 shows predictions and measure data for each surface length tested at $h=0.5$ (the first point acquired for all surface lengths off the nozzle lipline). The shortest surface $\left(x_{T E} / D_{e}=0.65\right)$ is already out of the jet flow by $h=0.5$ and the model correctly shows very little jet-surface interaction noise (Figure 12(a)). Extending the surface to $x_{T E} / D_{e}=1.35$ gives a small low frequency augmentation that is not reflected by the model but also shows the onset of a resonance in the mid-frequency range (Figure 12(b). These tones are amplified at $x_{T E} / D_{e}=2$ (Figure 12(c)) along with the low frequency jet-surface interaction noise and the model settles above the isolated jet noise but on the lower end of the jet-surface noise. The model improves for the longer surface at $x_{T E} / D_{e}=4$ but still misses at frequencies below the peak jet-surface interaction noise peak (Figure 12(d)). At first it appears the the peak amplitude coefficient determined by the model is just low but a closer examination shows that the measured spectra is not well defined as a symmetric parabola (look at the growing difference as frequency decreases) and the optimal model coefficients fit the higher frequencies better (although the peak amplitude may still be $1-2 \mathrm{~dB}$ low). This configuration presents a case for adding a linear term to the original parabolic model to provide finer control over the spectral shape (in exchange for another term, associated fits, coefficients, and general complexity). The model performs well at $x_{T E} / D_{e}=6$ (Figure $10(\mathrm{~b})$ ). The prediction at $x_{T E} / D_{e}=8$ shows a trend reversal from the shorter surfaces by slightly over estimating the peak amplitude as the measured spectra becomes very flat in the peak frequency region (Figure 12(e)). Finally, the predicted spectra at $x_{T E} / D_{e}=10$ gets close to the measured peak amplitude (Figure 12(f)) but with a wider spectral width (note lack of a defined peak at this position). It is important to remember that the jet-surface interaction noise across this range of surface lengths alone varies by approximately $20 \mathrm{~dB}$ (more when variations in $h$ are included) so while this model may lack the absolute accuracy often desired it does appear to fairly represent the trends in highly varying parameter space.

\section{IV.B. Velocity Scaling}

An empirical model for jet-surface interaction noise has been developed based on the idea that a characteristic spectra can be found for a range of surface positions at a single jet condition and then scaled to account for changes in jet velocity. Previous experimental and theoretical work has shown that jet-surface interaction noise is dipolar in nature and scales with velocity like $U_{e}^{5}$ to $U_{e}^{6}$ depending on primarily surface rigidity (e.g. ${ }^{4,5,7-9}$ ). The current jet-surface noise model has been implemented with $U_{e}^{6}$ velocity scaling. Figure 13 


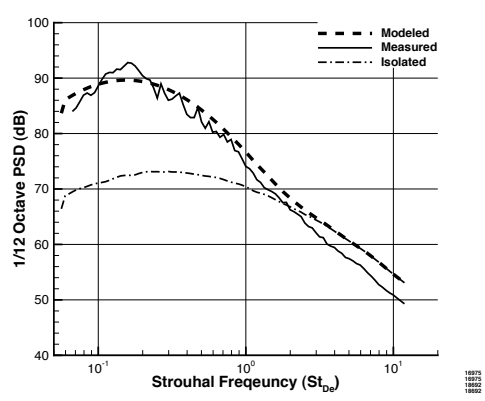

(a) $h=0.0$

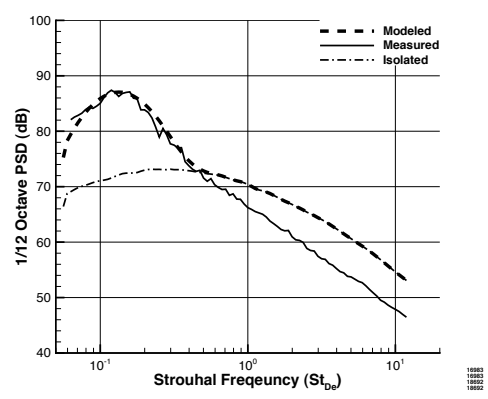

(d) $h=1.9$

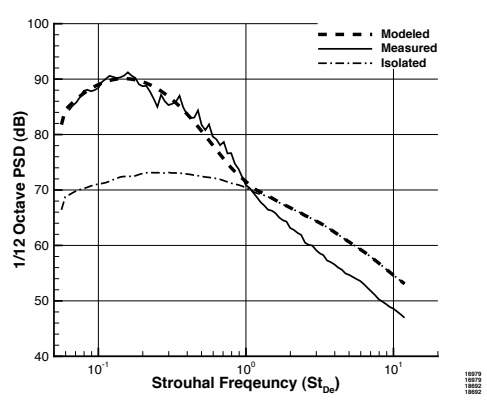

(b) $h=0.5$

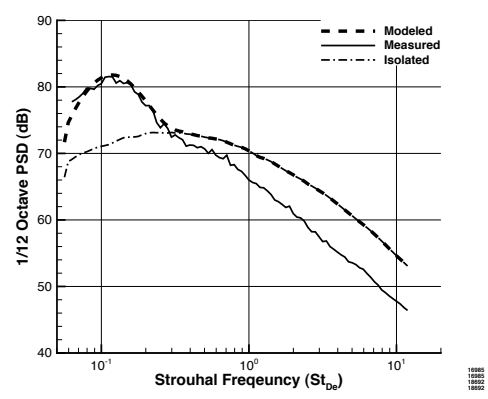

(e) $h=3.2$

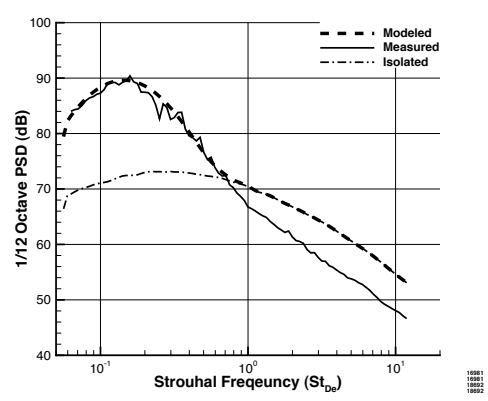

(c) $h=1.0$

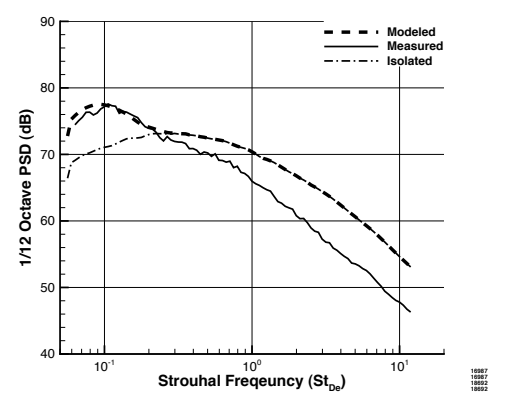

(f) $h=5.0$

Figure 10. Modeled spectra (dashed lines) compared to measured data (solid lines) for $x_{T E} / D_{e}=6 \mathrm{with}$ an unheated $M_{a}=0.5$ jet (setpoint 3 ).

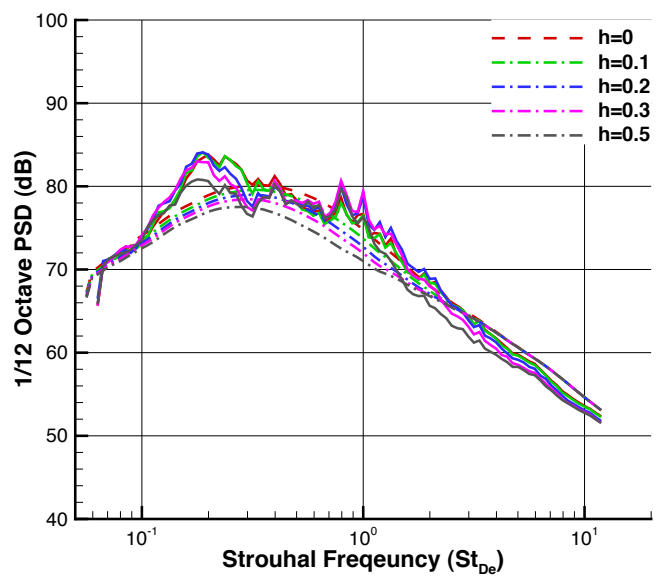

(a) $x_{T E} / D_{e}=2$

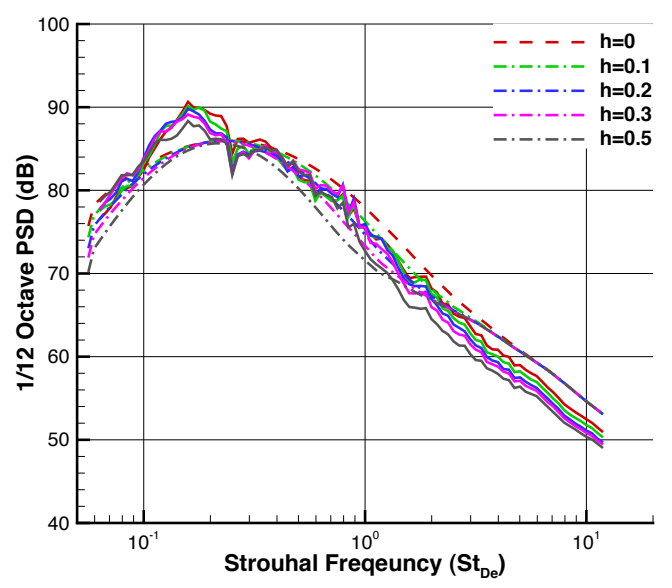

(b) $x_{T E} / D_{e}=4$

Figure 11. Comparison between modeled spectra (dashed lines) and measured data (solid lines) for an unheated $M_{a}=0.5$ unheated jet (setpoint 3 ) at surface positions close to the jet $(0 \leq h \leq 0.5)$. 


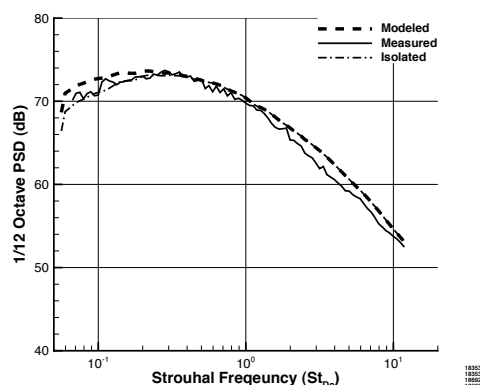

(a) $x_{T E} / D_{e}=0.65$

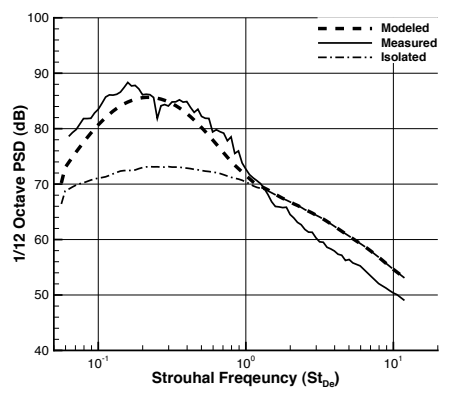

(d) $x_{T E} / D_{e}=4.0$

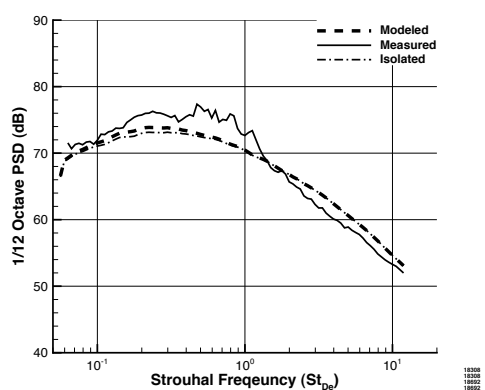

(b) $x_{T E} / D_{e}=1.35$

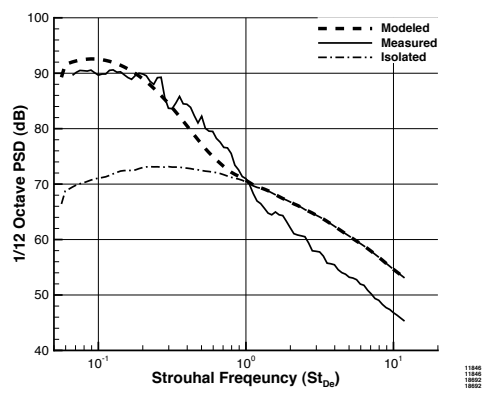

(e) $x_{T E} / D_{e}=8.0$

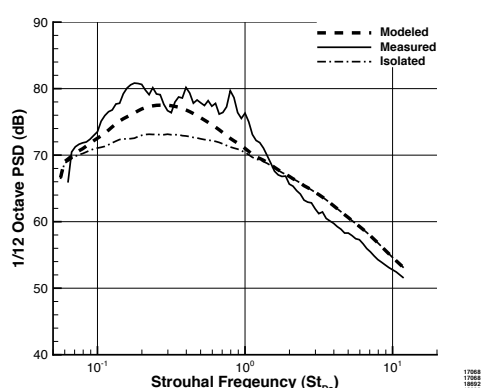

(c) $x_{T E} / D_{e}=2.0$

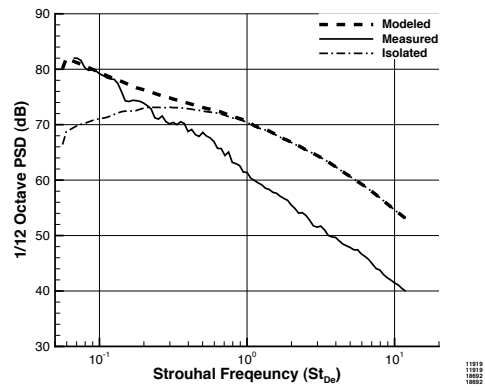

(f) $x_{T E} / D_{e}=10.0$

Figure 12. Modeled spectra (dashed lines) compared to measured data (solid lines) for $0.65 \leq x_{T E} / D_{e}=$ 10 at $h=0.5$ and an unheated $M_{a}=0.5$ jet (setpoint 3). Note that the comparison at $x_{T E} / D_{e}=6$ can be found in Figure 10(b) and so is not repeated here.

shows measured and modeled spectra for jet exit velocities $M_{a}=0.5, M_{a}=0.7$ and $M_{a}=0.9$ at $h=0.3$ (near the peak for jet-surface interaction noise at most surface lengths tested) and $0.65 \leq x_{T E} / D_{e} \leq 6$. When $x_{T E} / D_{e} \leq 2$, scaling the modeled characteristic spectra with $U_{e}^{6}$ fits the measured data well and, in fact, the fit improves as the resonance behavior found at $M_{a}=0.5$ disappears (or is masked by the jet mixing noise). At $x_{T E} / D_{e}=4$ and 6 , however, this velocity scaling breaks down around the jet-surface interaction noise peak frequency where the peak of the measured data at $M_{a}=0.7$ and 0.9 is flatter than at $M_{a}=0.5$. This change in spectral shape can not be accounted for by any velocity scaling rule without considering the scaling on a frequency by frequency basis; even then there may be issues as the simple scaling appears to hold for shorter surfaces requiring scaling based on frequency and surface length. Using the $U_{e}^{6}$ scaling introduces error up to $5 \mathrm{~dB}$ at $M_{a}^{9}$ and $x_{T E} / D_{e}=6$.

\section{IV.C. Directivity Scaling}

The empirical model developed to predict jet-surface interaction noise provides a characteristic spectra that can be scaled to account for changes in jet velocity or observer angle. Scaling the model spectra assuming that the jet-surface interaction noise source behaves like a pure dipole on a rigid plate was discussed in Section IV.B. These same assumption about the noise are now applied to describe changes in observer angle ${ }^{\mathrm{b}}$ Note that only polar angle, $\theta$, is considered here because the underlying dataset only includes measurements at one azimuthal angle. An extension to include dependence on azimuthal angle based on a recently acquired dataset is planned. Mathematically the dipole source at the trailing edge of the surface should change with observer angle $(\theta)$ appears as:

$$
P_{d}\left(x_{T E} / D_{e}, h, M_{a}, \theta\right)=P_{d}\left(x_{T E} / D_{e}, h, M_{a}, 90^{\circ}\right)\left[1-\cos ^{2}(\theta)\right]
$$

where $P_{d}$ is the modeled jet-surface interaction noise as a function of surface length $\left(x_{T E} / D_{e}\right)$, surface distance $(h)$, and Mach number $\left(M_{a}\right)$. The empirical model defines the characteristic spectra at $\theta=90^{\circ}$ where $P_{d}$ has its maximum value based on angle (i.e. $1-\cos ^{2}(\theta)=1$ ) so moving the observer angle upstream 


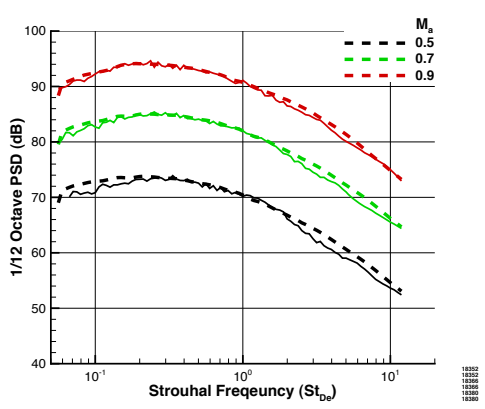

(a) $x_{T E} / D_{e}=0.65$

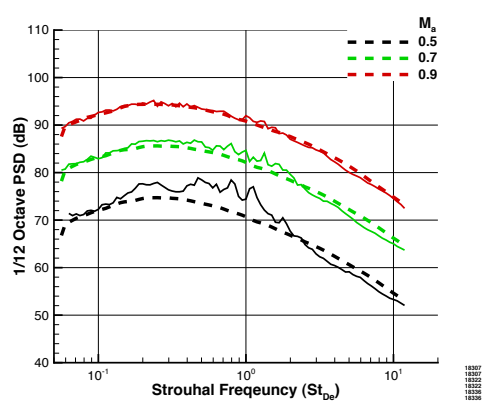

(b) $x_{T E} / D_{e}=1.35$

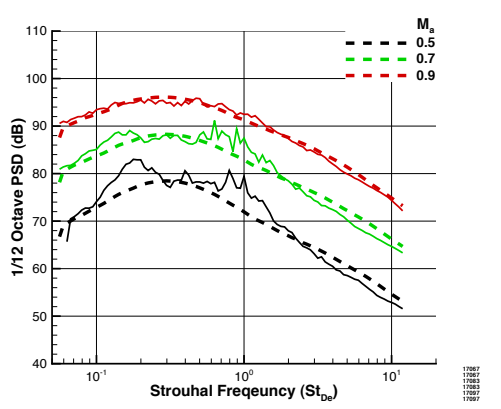

(c) $x_{T E} / D_{e}=2.0$

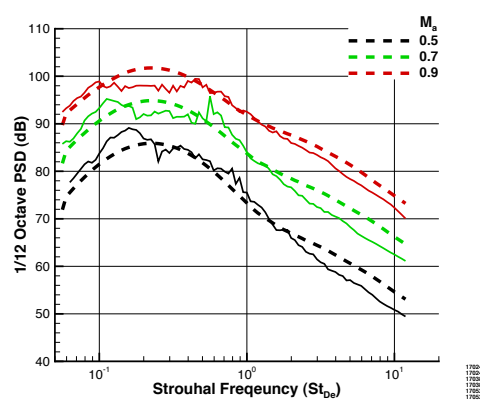

(d) $x_{T E} / D_{e}=4.0$

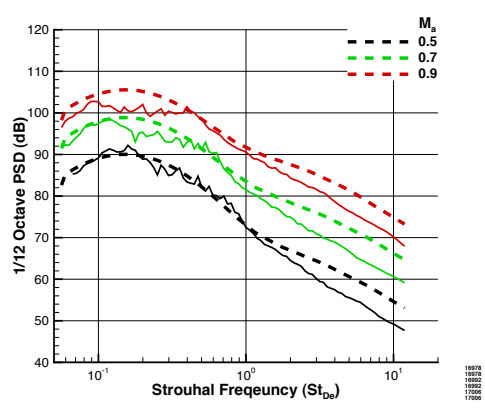

(e) $x_{T E} / D_{e}=6.0$

Figure 13. Modeled spectra (dashed lines) compared to measured data (solid lines) for $0.65 \leq x_{T E} / D_{e}=$ 10 at $h=0.3$ and an unheated $M_{a}=0.5, M_{a}=0.7$, and $M_{a}=0.9$ jets. Note that the deviations above $S t_{D e} \approx 1$ for the longer surfaces are due to the shielding of jet-mixing noise which is not included in the noise modeling. 
or downstream will reduce the predicted amplitude.

Two surface lengths will used to investigate the directivity of the reconstructed spectra. First, Figure 14 shows the shape of the reconstructed spectra, with contours representing the difference from the measured spectra, across all angles for the $x_{T E} / D_{e}=6$ surface and a range of $h$ locations. As shown in Section IV.A, the model performed fairly well compared to the experimental data at this surface length (see Figure 10) making it a good staring point for discussion of directivity scaling. At each surface distance $(h)$ in Figure 14 the spectra is dominated by a high ridge extending at relatively low frequency that is the jet-surface interaction noise. This ridge extends across most angles when the surface is close to the jet $h=0-0.5$ but fades into the jet mixing noise around $\theta \approx 120^{\circ}$ with the jet mixing noise dominating at downstream angles when the surface is far from the jet (e.g. Figure 14(f)). The largest errors appear in this cross-over region between $110 \leq \theta \leq 130$ with the worst case at $h=0.5$ and approximately $\theta=120^{\circ}$. Figure 15 shows a comparison between the modeled, measured, and isolated for this surface positions at $\theta=60^{\circ}, 90^{\circ}, 120^{\circ}$. At $\theta=60^{\circ}$ the jet-surface interaction noise peak is lower and flatter than predicted by the model (although it should be noted that this may partially be due to noise shielding at this angle) while there is good agreement at the characteristic spectra at $\theta=90^{\circ}$. However, at $\theta=120^{\circ}$ some additional noise appears past the jet-surface interaction noise peak that appears similar in character to the resonant noise observed in the characteristic spectra with shorter surfaces (Section IV.A). While it is not clear from these data if the noise at $\theta=120^{\circ}$ (and to a lesser extent at $\theta=150^{\circ}$ ) is generated by the same underlying mechanisms, it does account for most of the difference found Figure 14(f). Otherwise, this directivity scaling appears to track the peak amplitude of the jet-surface interaction noise fairly well.

The largest difference between the measured and modeled spectra for $x_{T E} / D_{e}=6$ appears has spectral characteristics that differ from the classic low frequency augmentation description of jet-surface interaction noise but are more like the resonant tones found in interaction noise created by a rectangular nozzle near a surface. ${ }^{1,13}$ Therefore, the $x_{T E} / D_{e}=2$ surface, which showed a significant amount of this type noise (Section IV.A), will be used as a second case to evaluate the directivity of the empirical model. Figure 16 compares the modeled and measured spectra at all angles for the $x_{T E} / D_{e}=2$ surface at several distances $(h)$. These data show a trend similar to that observed for $x_{T E} / D_{e}=6$; the peak differences are in the mid-angles $(100 \leq \theta \leq 130)$ at frequencies just above the peak frequency and are maximum at $h=0.5$. The absolute value of these difference is larger which would be consistent with the stronger resonance present in spectra from the shorter surface previously noted. Again extracting the spectra at $\theta=60^{\circ}, 90^{\circ}, 120^{\circ}$, and $150^{\circ}$ for a more detailed comparison, Figure 17 compares the modeled, measured, and isolated spectra for $x_{T E} / D_{e}=2$ and $h=0.5$. The model predicts the directivity change at $\theta=60^{\circ}$ fairly well. At $\theta=90^{\circ}$ and $\theta=120^{\circ}$, however, the differences are larger. In fact, the directivity model predicts that the jet-surface interaction noise should decrease between $\theta=90^{\circ}$ and $120^{\circ}$ but the peak spikes in the measured data are actually higher at $\theta=120^{\circ}$ (while the modeled spectra has decreased and is closer to the isolated spectra at $\theta=120^{\circ}$ than at $\theta=90^{\circ}$ ). These peaks appear to have a similar amplitudes at $\theta=90^{\circ}$ and $\theta=150^{\circ}$ but the model appears function better at the downstream angle largely because the increased jet mixing noise is more dominant. Thus, this aspect of the jet-surface interaction seems to follow a different directivity pattern than the more traditional low frequency augmentation and, combined with changes in this noise due to velocity noted in Section IV.B, would add considerable complexity to a relatively simple model. Alternatively, a more robust approach may be to build a second model dedicated only to tracking this more tonal noise and add it to the characteristic spectra, scaled for velocity and observer angle, produced by the parabolic model presented here. 


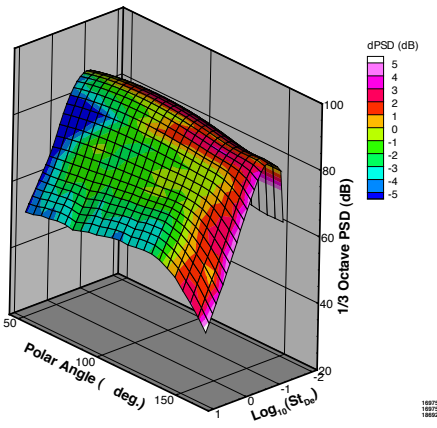

(a) $h=0.0$

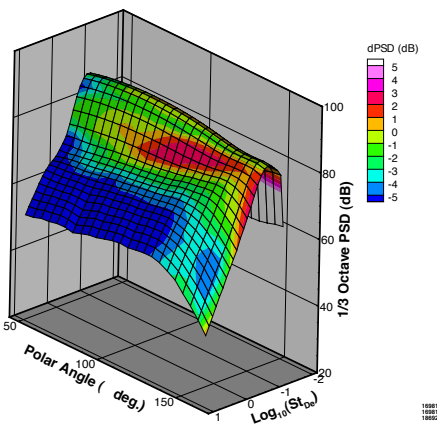

(d) $h=1.0$

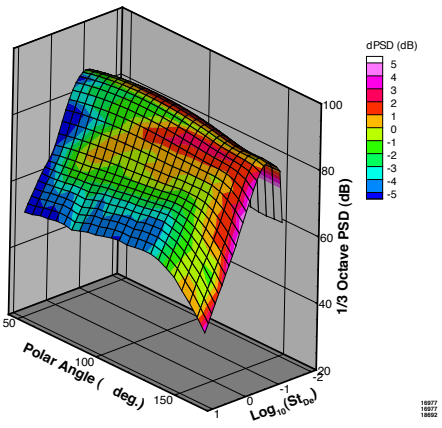

(b) $h=0.2$

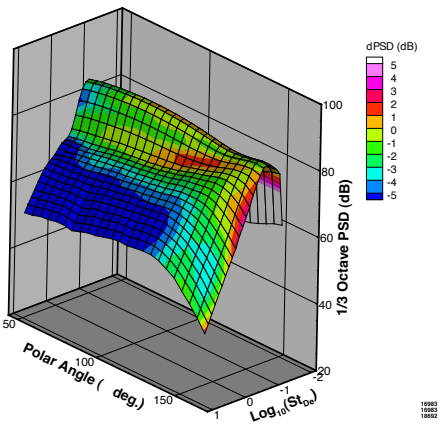

(e) $h=1.9$

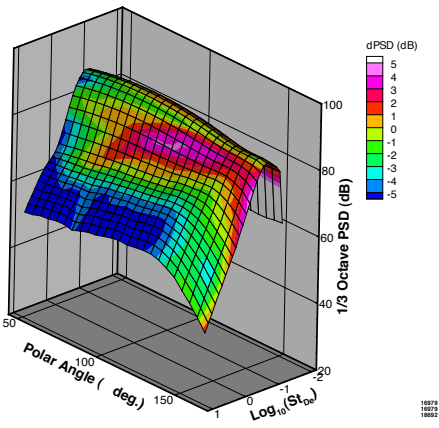

(c) $h=0.5$

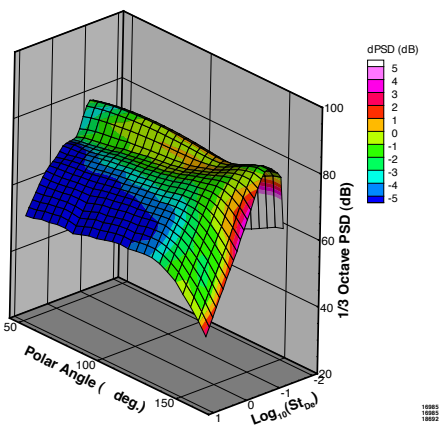

(f) $h=3.2$

Figure 14. Modeled spectra at all angles and frequencies with color contours showing $d P S D=$ $P S D_{\text {modeled }}-P S D_{\text {measured }}$ for $x_{T E} / D_{e}=6$ and $M_{a}=0.5$. Note that the dark blue region starting at upstream angles and high frequencies and extending out as the $h$ increases is caused by the surface shielding the jet mixing noise in the experimental data.

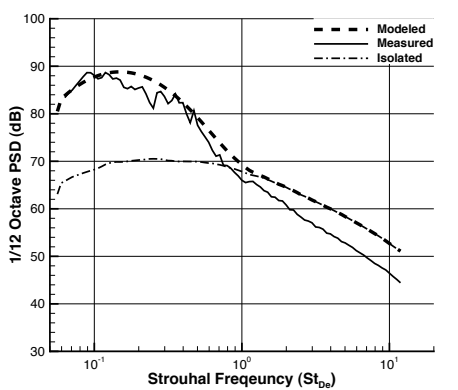

(a) $\theta=60^{\circ}$

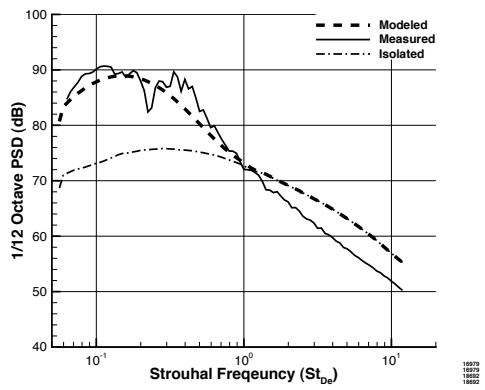

(c) $\theta=120^{\circ}$

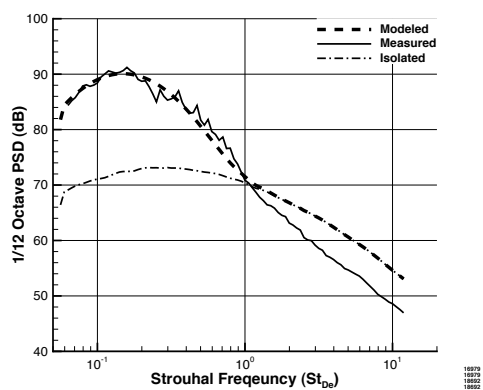

(b) $\theta=90^{\circ}$

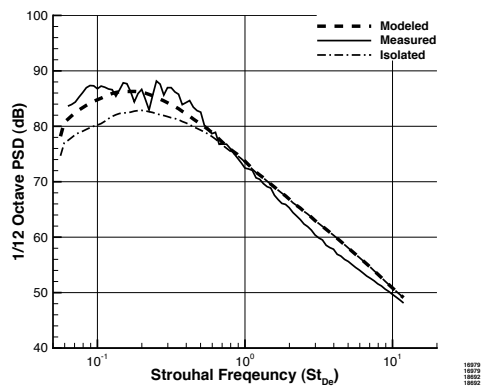

(d) $\theta=150^{\circ}$

Figure 15. Modeled, measured and isolated spectra at four polar angles for a $x_{T E} / D_{e}=6$ surface at $h=0.5$ and jet velocity $M_{a}=0.5$. 


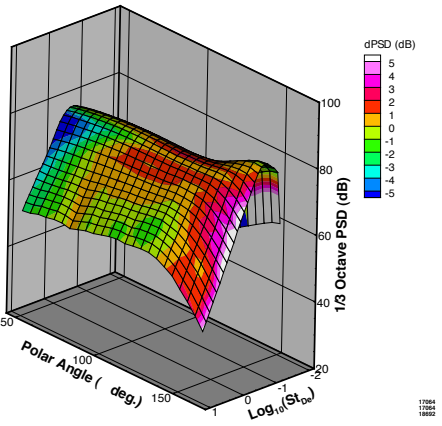

(a) $h=0.0$

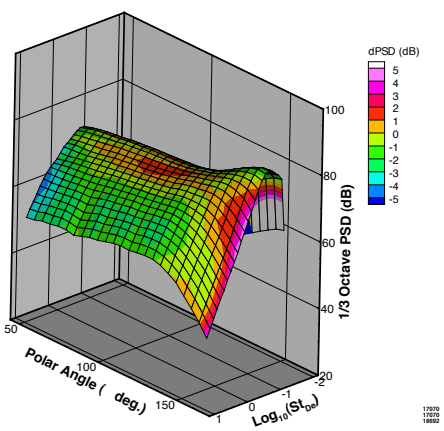

(d) $h=1.0$

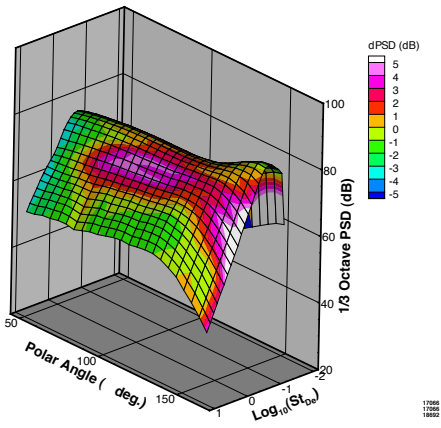

(b) $h=0.2$

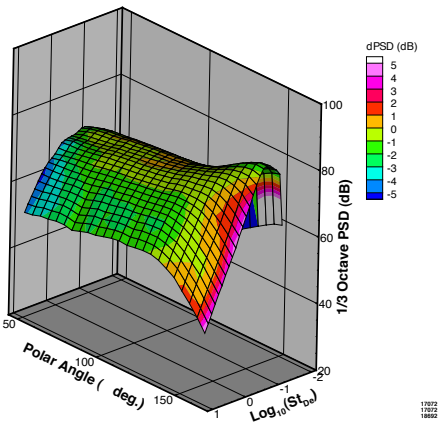

(e) $h=1.9$

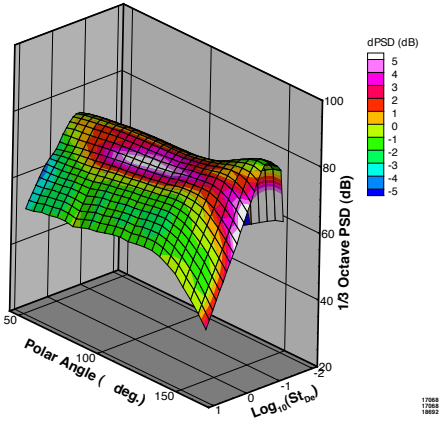

(c) $h=0.5$

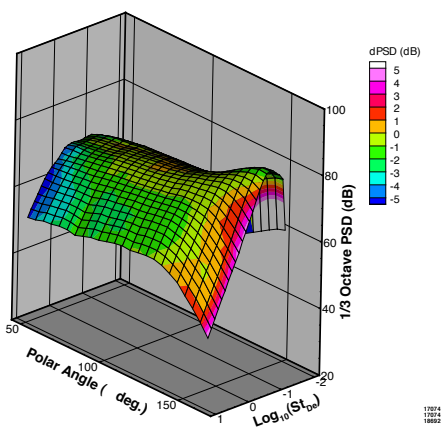

(f) $h=3.2$

Figure 16. Modeled spectra at all angles and frequencies with color contours showing $d P S D=$ $P S D_{\text {modeled }}-P S D_{\text {measured }}$ for $x_{T E} / D_{e}=2$ and $M_{a}=0.5$.

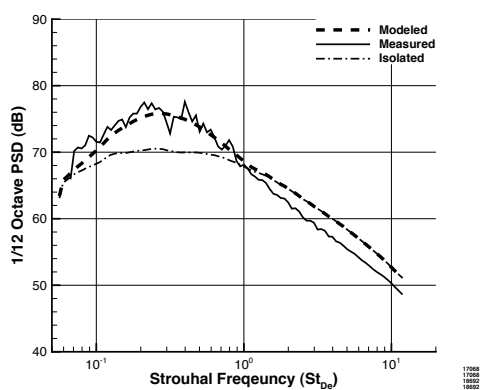

(a) $\theta=60^{\circ}$

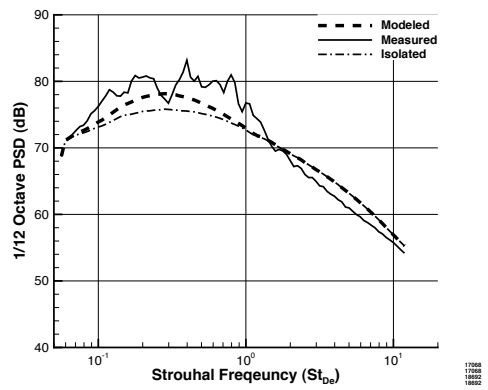

(c) $\theta=120^{\circ}$

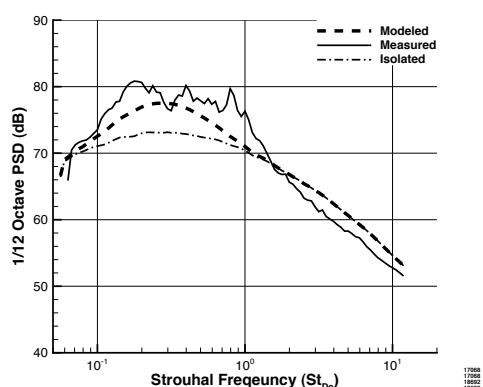

(b) $\theta=90^{\circ}$

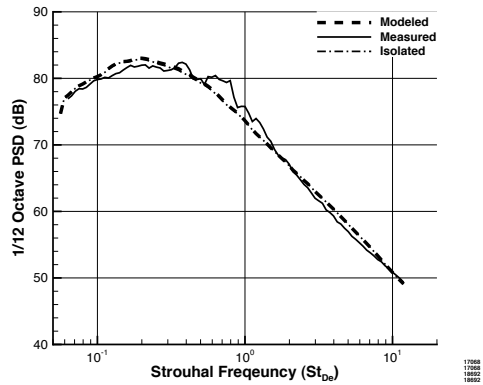

(d) $\theta=150^{\circ}$

Figure 17. Modeled, measured and isolated spectra at four polar angles for a $x_{T E} / D_{e}=2$ surface at $h=0.5$ and jet velocity $M_{a}=0.5$. 


\section{Conclusions}

The process of developing an empirical model to predict noise created by a jet near a surface has been described and the resulting model presented. This model attempts to represent the complete jet-surface interaction noise spectra by capturing trends in peak amplitude, spectral width, and peak frequency as functions of surface length and distance to produce a parabolic characteristic spectra which can then be scaled to represent different jet Mach numbers and observer angles. The model is relatively simple in design which allows for the addition of other models to increase the range of applicability (e.g. heated jets, rectangular nozzles) or improve the accuracy by incorporating spectral details not easily modeled as part of the larger system (e.g. jet-surface resonances). An comparison between the model results and measured data showed several areas where the model could be improved if some additional complexity were added such as including a linear term in the parabolic model to account for non-symmetric spectra. In its current form this empirical model is best suited for preliminary system level design studies where computation speed is valued over absolute accuracy and the goal is to select potential configurations for more detailed study.

Subsonic and supersonic aircraft designs are moving to more integrated engine/airframe configurations in response to for greater efficiency and lower noise. Aircraft noise prediction tools are currently lacking to ability to quickly produce noise estimates for these tightly integrated configurations. The model presented represents a first step toward developing a more advanced capability in this area that can be used to evaluate future aircraft designs.

\section{References}

${ }^{1}$ Bridges, J., "Noise from Aft Deck Exhaust Nozzles - Differences in Experimental Embodiments", to be presented at the 2014 AIAA SciTech Conference, 13-17 January, 2014, National Harbor, MD, AIAA 2014-????.

${ }^{2}$ Brown, C. and Bridges, J., "Small Hot Jet Acoustic Rig Validation", NASA/TM-2006-214324, 2006.

${ }^{3}$ Bridges, J. and Brown, C., "Validation of the Small Hot Jet Acoustic Rig for Jet Noise Research", AIAA 2005-2846, 2005. 1955.

${ }^{4}$ Curle, N., "The influence of solid boundaries upon aerodynamic sound", Proc. Roy. Soc. of London, 231A, pp. 505-514,

${ }^{5}$ Ffowcs-Williams, J.E. and Hall, L.H., "Aerodynamic sound generation by turbulent flow in the vicinity of a scattering half plane", J. Fluid Mech., 40(4), pp. 657-670, 1970.

${ }^{6}$ Crighton, D.G. and Leppington, F.G., "Scattering of aerodynamic noise by a semi-infinite compliant plate", J. Fluid Mech., 43(4), pp. 721-736, 1970.

${ }^{7}$ Head, R.W. and Fisher, M.J., "Jet/Surface Interaction Noise: Analysis Of Farfield Low Frequency Augmentation of Jet Noise Due To The Presence Of A Solid Surface", AIAA 1976-502, 1976.

${ }^{8}$ Lawrence, J.L.T., Azarpeyvand, M., and Self, R.H., "Interaction between a Flat Plate and a Circular Jet", AIAA 2011$2745,2011$.

${ }^{9}$ Brown, C.A. , "Jet-Surface Interaction Test: Far-Field Noise Results", J. Eng. Gas Turbines Power, 135(7), Jun. 2013.

${ }^{10}$ Podboy, G., "Jet-Surface Interaction Test: Phased Array Noise Source Localization Results", ASME GT2012-69801, 2012.

${ }^{11}$ Khavaran, A. and Bridges, J., "Development of jet noise power spectral laws using SHJAR data", AIAA 209-3378, 2009.

${ }^{12}$ Khavaran, A. and Bridges, J., "SHJAR jet noise data and power spectral laws", NASA/TM 2009-215608, 2009.

${ }^{13}$ Zaman, K.B.M.Q., Fagan, A.F., Clem, M.M., and Brown, C.A., "Resonant Interaction of a Rectangular Jet with a Flatplate", to be presented at the 2014 AIAA SciTech Conference, 13-17 January, 2014, National Harbor, MD., AIAA 2014-????.

\section{Appendix A. Equations and Coefficients for $A\left(x_{T E} / D_{e}, h\right)$ Fit}

The variable $A\left(x_{T E} / D_{e}, h\right)$ in the dipole noise source model (Section III.B, Equation 1 ) is given by the equation:

$$
A\left(x_{T E} / D_{e}, h\right)=A_{x^{3}}\left(x_{T E} / D_{e}\right) h^{3}+A_{x^{2}}\left(x_{T E} / D_{e}\right) h^{2}+A_{x^{1}}\left(x_{T E} / D_{e}\right) h^{1}+A_{x^{0}}\left(x_{T E} / D_{e}\right)
$$

where the values of $A_{x^{3}}\left(x_{T E} / D_{e}\right), A_{x^{2}}\left(x_{T E} / D_{e}\right), A_{x^{1}}\left(x_{T E} / D_{e}\right)$, and $A_{x^{0}}\left(x_{T E} / D_{e}\right)$ are given by the results of the fit process described in Section III.B.1. $A_{x^{3}}, A_{x^{2}}$ and $A_{x^{1}}$ were fit using the cubic polynomial: 


$$
A_{x^{n}}\left(x_{T E} / D_{e}\right)=\left(x^{3}\right)\left(x_{T E} / D_{e}\right)^{3}+\left(x^{2}\right)\left(x_{T E} / D_{e}\right)^{2}+\left(x^{1}\right)\left(x_{T E} / D_{e}\right)^{1}+\left(x^{0}\right)\left(x_{T E} / D_{e}\right)^{0}
$$

where values of $\left(x^{n}\right)$ are provided in Tables 3,4 , and 5 (with the appropriate range of applicability). The value of $A_{x^{0}}$ is obtained from a logarithmic equation:

$$
A_{x^{0}}\left(x_{T E} / D_{e}\right)=9.3392 \ln \left(x_{T E} / D_{e}\right)+72.821
$$

which is valid across the range $1 \leq x_{T E} / D_{e} \leq 10$.

\begin{tabular}{|c|c|c|c|c|}
\hline Range & $x^{3}$ & $x^{2}$ & $x^{1}$ & $x^{0}$ \\
\hline $0.5 \leq x_{T E} / D_{e}<1.35$ & 0 & 0 & -0.4436 & 0.372 \\
\hline $1.35 \leq x_{T E} / D_{e}<4$ & -0.001648 & -0.00348 & 0.231256 & -0.538605 \\
\hline $4 \leq x_{T E} / D_{e} \leq 10$ & -0.012032 & 0.219824 & -1.288074 & 2.6955 \\
\hline
\end{tabular}

Table 3. Coefficients for computing the value of $A_{x^{3}}\left(x_{T E} / D_{e}\right)$ in Equation 8 (based on a cubic polynomial). Note that the unusually large number of significant figures in the coefficients are needed here because of the potential large values of $\left(x_{T E} / D_{e}\right)^{3}$.

\begin{tabular}{|c|c|c|c|c|}
\hline Range & $x^{3}$ & $x^{2}$ & $x^{1}$ & $x^{0}$ \\
\hline $0.5 \leq x_{T E} / D_{e} \leq 1.35$ & 0 & -3.6084 & 11.4422 & -6.6087 \\
\hline $1.35<x_{T E} / D_{e}<8$ & 0 & 0 & -1.9986 & 5.8391 \\
\hline $8 \leq x_{T E} / D_{e} \leq 10$ & 0.066408 & -1.139036 & 6.249492 & -13.1788 \\
\hline
\end{tabular}

Table 4. Coefficients for computing the value of $A_{x^{2}}\left(x_{T E} / D_{e}\right)$ in Equation 8 (based on a cubic polynomial).

\begin{tabular}{|c|c|c|c|c|}
\hline Range & $x^{3}$ & $x^{2}$ & $x^{1}$ & $x^{0}$ \\
\hline $0.5 \leq x_{T E} / D_{e}<2$ & 0 & 7.512 & -27.178 & 16.029 \\
\hline $2 \leq x_{T E} / D_{e}<8$ & 0.097432 & -2.026624 & 13.730666 & -28.4122 \\
\hline $8 \leq x_{T E} / D_{e} \leq 10$ & 0 & 0 & -3.2284 & 27.442 \\
\hline
\end{tabular}

Table 5. Coefficients for computing the value of $A_{x^{1}}\left(x_{T E} / D_{e}\right)$ in Equation 8 (based on a cubic polynomial).

\section{Appendix B. Equations and Coefficients for $B\left(x_{T E} / D_{e}, h\right)$ Fit}

The variable $B\left(x_{T E} / D_{e}, h\right)$ in the dipole noise source model (Section III.B, Equation 1) is given by the equation:

$$
B\left(x_{T E} / D_{e}, h\right)=B_{x^{3}}\left(x_{T E} / D_{e}\right) h^{3}+B_{x^{2}}\left(x_{T E} / D_{e}\right) h^{2}+B_{x^{1}}\left(x_{T E} / D_{e}\right) h^{1}+B_{x^{0}}\left(x_{T E} / D_{e}\right)
$$

where the values of $B_{x^{3}}, B_{x^{2}}, B_{x^{1}}$, and $B_{x^{0}}$ are given by the results of the fit process described in Section III.B.2. $B_{x^{3}}, B_{x^{2}}, B_{x^{1}}$, and $B_{x^{0}}$ were fit using the cubic polynomial:

$$
B_{x^{n}}\left(x_{T E} / D_{e}\right)=\left(x^{3}\right)\left(x_{T E} / D_{e}\right)^{3}+\left(x^{2}\right)\left(x_{T E} / D_{e}\right)^{2}+\left(x^{1}\right)\left(x_{T E} / D_{e}\right)^{1}+\left(x^{0}\right)\left(x_{T E} / D_{e}\right)^{0}
$$

where $\left(x^{3}\right),\left(x^{2}\right),\left(x^{1}\right)$, and $\left(x^{0}\right)$ are the coefficients corresponding to the cubic, quadratic, linear, and constant terms respectively. These coefficients (with the appropriate range of applicability) are provided in Tables 6 , 7,8 , and 9. 


\begin{tabular}{|c|c|c|c|c|}
\hline Range & $x^{3}$ & $x^{2}$ & $x^{1}$ & $x^{0}$ \\
\hline $0.5 \leq x_{T E} / D_{e}<2$ & 0 & 0.2992 & 0.4038 & -2.4129 \\
\hline $2 \leq x_{T E} / D_{e}<4$ & 0 & 0.142324 & -0.80865 & 0.6394 \\
\hline $4 \leq x_{T E} / D_{e}<10$ & 0.074472 & -1.687588 & 11.83085 & -25.406 \\
\hline
\end{tabular}

Table 6. Coefficients for computing the value of $B_{x^{3}}\left(x_{T E} / D_{e}\right)$ in Equation 11 (based on a cubic polynomial).

\begin{tabular}{|c|c|c|c|c|}
\hline Range & $x^{3}$ & $x^{2}$ & $x^{1}$ & $x^{0}$ \\
\hline $0.5 \leq x_{T E} / D_{e}<2$ & 0 & 2.3888 & -14.0346 & 26.689 \\
\hline $2 \leq x_{T E} / D_{e}<4$ & 0 & -1.871788 & 12.816652 & -9.9713 \\
\hline $4 \leq x_{T E} / D_{e}<6$ & -0.65556 & 14.626664 & -102.345184 & 228.657 \\
\hline $6 \leq x_{T E} / D_{e}<10$ & 0 & -1.106788 & 20.900176 & -86.012 \\
\hline
\end{tabular}

Table 7. Coefficients for computing the value of $B_{x^{2}}\left(x_{T E} / D_{e}\right)$ in Equation 11 (based on a cubic polynomial).

\begin{tabular}{|c|c|c|c|c|}
\hline Range & $x^{3}$ & $x^{2}$ & $x^{1}$ & $x^{0}$ \\
\hline $0.5 \leq x_{T E} / D_{e}<2$ & 0 & -19.26284 & 63.522824 & -82.938286 \\
\hline $2 \leq x_{T E} / D_{e}<6$ & 0 & 5.4808 & -43.334 & 31.801 \\
\hline $6 \leq x_{T E} / D_{e}<10$ & 0 & 1.252 & -18.9752 & 37.882 \\
\hline
\end{tabular}

Table 8. Coefficients for computing the value of $B_{x^{1}}\left(x_{T E} / D_{e}\right)$ in Equation 11 (based on a cubic polynomial).

\begin{tabular}{|c|c|c|c|c|}
\hline Range & $x^{3}$ & $x^{2}$ & $x^{1}$ & $x^{0}$ \\
\hline $0.5 \leq x_{T E} / D_{e}<4$ & 0 & 6.3312 & -34.4 & 14.926 \\
\hline $4 \leq x_{T E} / D_{e}<10$ & 0 & 0.4356 & -3.965 & -12.804 \\
\hline $0 \leq x_{T E} / D_{e}<0$ & 0 & 0 & 0 & 0 \\
\hline
\end{tabular}

Table 9. Coefficients for computing the value of $B_{x^{0}}\left(x_{T E} / D_{e}\right)$ in Equation 11 (based on a cubic polynomial). 


\section{Appendix C. Equations and Coefficients for $F_{p e a k}\left(x_{T E} / D_{e}, h\right)$ Fit}

The variable $F_{\text {peak }}\left(x_{T E} / D_{e}, h\right)$ in the dipole noise source model (Section III.B, Equation 1) is given by the equation:

$$
F_{\text {peak }}\left(x_{T E} / D_{e}, h\right)=F_{x^{2}}\left(x_{T E} / D_{e}\right) h^{2}+F_{x^{1}}\left(x_{T E} / D_{e}\right) h^{1}+F_{x^{0}}\left(x_{T E} / D_{e}\right)
$$

where the values of $F_{x^{3}}, F_{x^{2}}, F_{x^{1}}$, and $F_{x^{0}}$ are given by the results of the fit process described in Section III.B.3. $F_{x^{3}}, F_{x^{2}}, F_{x^{1}}$, and $F_{x^{0}}$ were fit using the cubic polynomial:

$$
F_{x^{n}}\left(x_{T E} / D_{e}\right)=\left(x^{3}\right)\left(x_{T E} / D_{e}\right)^{3}+\left(x^{2}\right)\left(x_{T E} / D_{e}\right)^{2}+\left(x^{1}\right)\left(x_{T E} / D_{e}\right)^{1}+\left(x^{0}\right)\left(x_{T E} / D_{e}\right)^{0}
$$

where $\left(x^{3}\right),\left(x^{2}\right),\left(x^{1}\right)$, and $\left(x^{0}\right)$ are the coefficients corresponding to the cubic, quadratic, linear, and constant terms respectively. These coefficients (with the appropriate range of applicability) are provided in Tables 10,11 , and 12 .

\begin{tabular}{|c|c|c|c|c|}
\hline Range & $x^{3}$ & $x^{2}$ & $x^{1}$ & $x^{0}$ \\
\hline $0.5 \leq x_{T E} / D_{e}<2$ & 0 & -0.024 & 0.08 & -0.045 \\
\hline $2 \leq x_{T E} / D_{e}<10$ & -0.00020833336 & 0.0037 & -0.02356666 & 0.0525 \\
\hline
\end{tabular}

Table 10. Coefficients for the cubic polynomial used to compute the value of $F_{x^{2}}\left(x_{T E} / D_{e}\right)$ in Equation 13.

\begin{tabular}{|c|c|c|c|c|}
\hline Range & $x^{3}$ & $x^{2}$ & $x^{1}$ & $x^{0}$ \\
\hline $0.5 \leq x_{T E} / D_{e}<2$ & 0 & 0.1432 & -0.4952 & 0.2824 \\
\hline $2 \leq x_{T E} / D_{e}<10$ & 0.000944 & -0.01882 & 0.132992 & -0.33358 \\
\hline
\end{tabular}

Table 11. Coefficients for the cubic polynomial used to compute the value of $F_{x^{1}}\left(x_{T E} / D_{e}\right)$ in Equation 13.

\begin{tabular}{|c|c|c|c|c|}
\hline Range & $x^{3}$ & $x^{2}$ & $x^{1}$ & $x^{0}$ \\
\hline $0.5 \leq x_{T E} / D_{e}<2$ & 0 & -0.1412 & 0.5734 & -0.2321 \\
\hline $2 \leq x_{T E} / D_{e}<10$ & 0 & 0.0023 & -0.0652 & 0.46176 \\
\hline
\end{tabular}

Table 12. Coefficients for the cubic polynomial used to compute the value of $F_{x^{0}}\left(x_{T E} / D_{e}\right)$ in Equation 13. 



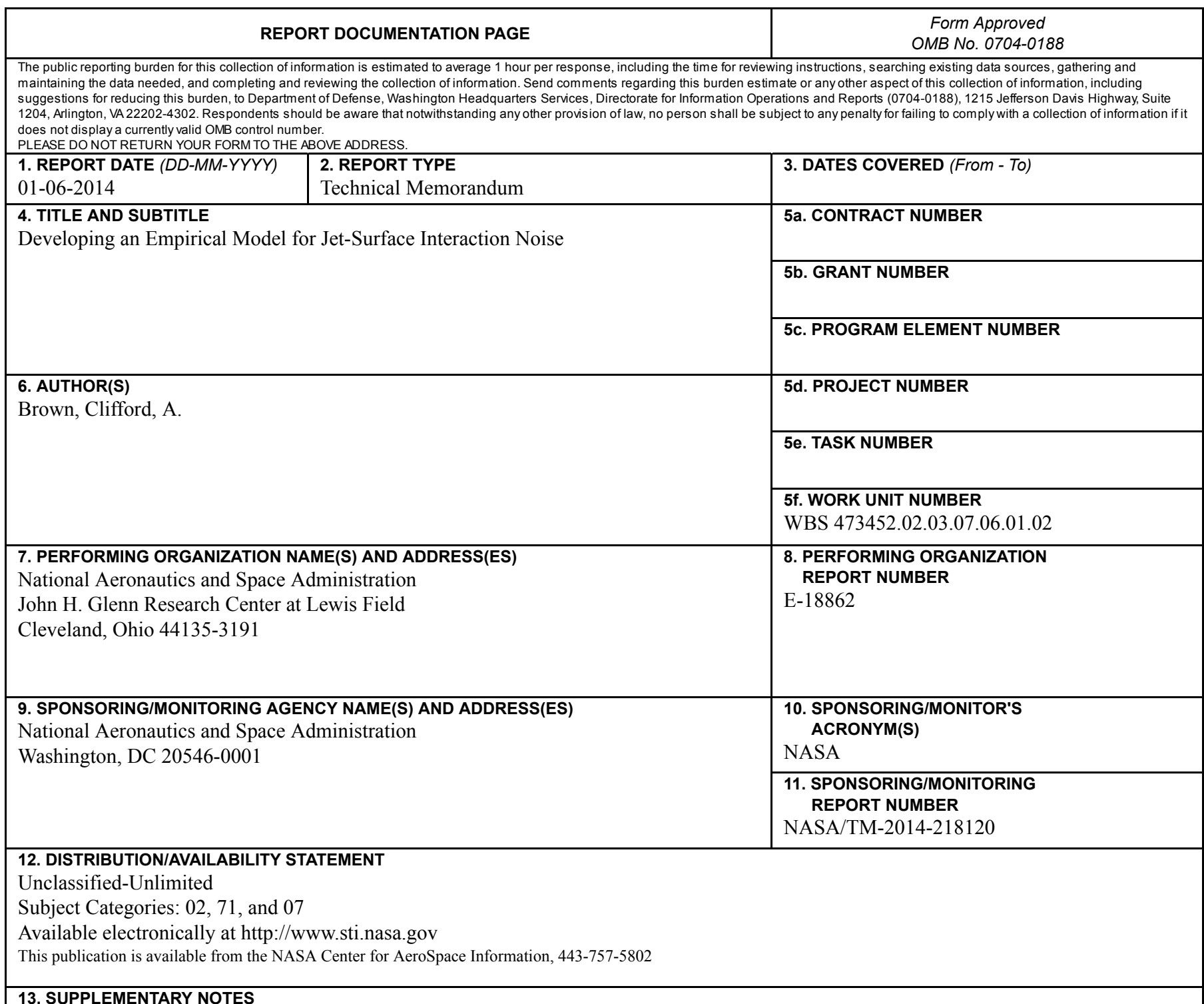

\section{SUPPLEMENTARY NOTES}

\section{ABSTRACT}

The process of developing an empirical model for jet-surface interaction noise is described and the resulting model evaluated. Jet-surface interaction noise is generated when the high-speed engine exhaust from modern tightly integrated or conventional high-bypass ratio engine aircraft strikes or flows over the airframe surfaces. An empirical model based on an existing experimental database is developed for use in preliminary design system level studies where computation speed and range of configurations is valued over absolute accuracy to select the most promising (or eliminate the worst) possible designs. The model developed assumes that the jet-surface interaction noise spectra can be separated from the jet mixing noise and described as a parabolic function with three coefficients: peak amplitude, spectral width, and peak frequency. These coefficients are fit to functions of surface length and distance from the jet lipline to form a characteristic spectra which is then adjusted for changes in jet velocity and/or observer angle using scaling laws from published theoretical and experimental work. The resulting model is then evaluated for its ability to reproduce the characteristic spectra and then for reproducing spectra measured at other jet velocities and observer angles; successes and limitations are discussed considering the complexity of the jet-surface interaction noise versus the desire for a model that is simple to implement and quick to execute.

\section{SUBJECT TERMS}

Aerodynamic noise; (airframe generated); Aerodynamic noise (general); Aerodynamic noise (propulsion systems); Jet engines; Engine noise

\begin{tabular}{|c|c|c|c|c|c|}
\hline \multicolumn{3}{|c|}{ 16. SECURITY CLASSIFICATION OF: } & \multirow{3}{*}{$\begin{array}{l}\text { 17. LIMITATION OF } \\
\text { ABSTRACT } \\
\text { UU }\end{array}$} & \multirow{3}{*}{$\begin{array}{l}\text { 18. NUMBER } \\
\text { OF } \\
\text { PAGES } \\
32\end{array}$} & \multirow{2}{*}{$\begin{array}{l}\text { 19a. NAME OF RESPONSIBLE PERSON } \\
\text { STI Help Desk (email:help@sti.nasa.gov) }\end{array}$} \\
\hline a. REPORT & b. ABSTRACT & c. THIS PAGE & & & \\
\hline & & & & & $\begin{array}{l}\text { 19b. TELEPHONE NUMBER (include area code) } \\
443-757-5802\end{array}$ \\
\hline
\end{tabular}



